\title{
Volatiles Produced by Yeasts Related to Prunus avium and $P$. cerasus Fruits and Their Potentials to Modulate the Behaviour of the Pest Rhagoletis cerasi Fruit Flies
}

\author{
Raimondas Mozūraitis ${ }^{1}$ *(D), Violeta Apšegaitė ${ }^{1}$, Sandra Radžiutè ${ }^{1}$, Dominykas Aleknavičius ${ }^{1}$, Jurga Būdiené ${ }^{1}$, \\ Ramunè Stanevičiené ${ }^{2}$, Laima Blažytè-Čereškiené ${ }^{1}$, Elena Serviené ${ }^{2} \mathbb{D}$ and Vincas Būda ${ }^{1}$ \\ 1 Laboratory of Chemical and Behavioural Ecology, Institute of Ecology, Nature Research Centre, \\ Akademijos Str. 2, LT-08412 Vilnius, Lithuania; violeta.apsegaite@gamtc.lt (V.A.); \\ sandra.radziute@gamtc.lt (S.R.); dominykas.aleknavicius@gamtc.lt (D.A.); jurga.budiene@gamtc.lt (J.B.); \\ laima.blazyte@gamtc.lt (L.B.-Č.); vincas.buda@gamtc.lt (V.B.) \\ 2 Laboratory of Genetics, Institute of Botany, Nature Research Centre, Akademijos Str. 2, \\ LT-08412 Vilnius, Lithuania; ramune.staneviciene@gamtc.lt (R.S.); elena.serviene@gamtc.lt (E.S.) \\ * Correspondence: raimondas.mozuraitis@gamtc.lt; Tel.: +370-5-272-92-42
}

Citation: Mozūraitis, R.; Apšegaitè, V.; Radžiutè, S.; Aleknavičius, D.;

Būdienè, J.; Stanevičienè, R.; Blažytė-Čereškienė, L.; Servienè, E.; Būda, V. Volatiles Produced by Yeasts Related to Prunus avium and P. cerasus Fruits and Their Potentials to Modulate the Behaviour of the Pest Rhagoletis cerasi Fruit Flies. J. Fungi 2022, 8, 95. https://doi.org/ $10.3390 /$ jof 8020095

Academic Editor: Laurent Dufossé

Received: 9 December 2021

Accepted: 6 January 2022

Published: 19 January 2022

Publisher's Note: MDPI stays neutral with regard to jurisdictional claims in published maps and institutional affiliations.

Copyright: (C) 2022 by the authors. Licensee MDPI, Basel, Switzerland. This article is an open access article distributed under the terms and conditions of the Creative Commons Attribution (CC BY) license (https:// creativecommons.org/licenses/by/ $4.0 /)$

\begin{abstract}
Yeast produced semiochemicals are increasingly used in pest management programs, however, little is known on which yeasts populate cherry fruits and no information is available on the volatiles that modify the behaviour of cherry pests including Rhagoletis cerasi flies. Eighty-two compounds were extracted from the headspaces of eleven yeast species associated with sweet and sour cherry fruits by solid phase micro extraction. Esters and alcohols were the most abundant volatiles released by yeasts. The multidimensional scaling analysis revealed that the odour blends emitted by yeasts were species-specific. Pichia kudriavzevii and Hanseniaspora uvarum yeasts released the most similar volatile blends while P. kluyveri and Cryptococcus wieringae yeasts produced the most different blends. Combined gas chromatographic and electroantennographic detection methods showed that 3-methybutyl acetate, 3-methylbutyl propionate, 2-methyl-1-butanol, and 3-methyl-1butanol elicited antennal responses of both $R$. cerasi fruit fly sexes. The two-choice olfactometric tests revealed that $R$. cerasi flies preferred 3-methylbutyl propionate and 3-methyl-1-butanol but avoided 3-methybutyl acetate. Yeast-produced behaviourally active compounds indicated a potential for use in pest monitoring and control of $R$. cerasi fruit flies, an economically important pest of cherry fruits.
\end{abstract}

Keywords: yeasts; microorganisms; Diptera; Tephritidae; volatiles; attractant; repellent; electroantennography; pest management; behaviour modification

\section{Introduction}

Carposphere is a specific habitat populated by bacterial and fungal microorganisms including yeasts [1,2]. Berries and fruits are rich in carbohydrates and often bear the most diverse microbiome in a phyllosphere [2]. Carposphere microbiota are determined by a variety of factors such as environmental conditions, host genotype, berry developmental stage, and interactions with other organisms sharing a habitat [3-7]. Microorganisms associated with fruits and berries interact with insects that use these habitats for feeding and oviposition. Insects and yeasts could come into diverse relationships ranging from amensal to commensal and mutualistic [8]. Yeasts provide essential nutrients missing in sugar-rich berries that insects cannot produce while insects transfer yeasts from one substrate to another $[1,9,10]$. In addition to berry and fruit-related odours, yeast-produced volatiles are used by insects to acquire information about habitat quality and host choice [11-14]. The behaviour modifying effect of yeast volatiles has the potential for use in integrated pest management programs increasing the efficiency of attractive lures and serving as repellents in the push-pull pest control strategy [15]. 
The purpose of the study was to characterize volatile blends emitted by cultivable yeasts populating the fruit surface of sweet and sour cherries and to determine semiochemicals that modify the behaviour of $R$. cerasi fruit flies, the most important pest of cherry fruits.

\section{Materials and Methods}

\subsection{Yeast Sampling, Culturing, and Identification}

Yeast species have been isolated from sweet cherry (Prunus avium L.) and sour cherry (Prunus cerasus L.) (Rosales: Rosaceae) fruits collected during June-July of 2018-2020 from private plantations located in the Vilnius region (GPS coordinates: $54^{\circ} 45^{\prime} 08.2^{\prime \prime} \mathrm{N}$, $25^{\circ} 17^{\prime} 10.0^{\prime \prime} \mathrm{E} ; 54^{\circ} 46^{\prime} 14.4^{\prime \prime} \mathrm{N} 25^{\circ} 21^{\prime} 04.1^{\prime \prime} \mathrm{E} ; 54^{\circ} 41^{\prime} 19.9^{\prime \prime} \mathrm{N} 25^{\circ} 26^{\prime} 20.6^{\prime \prime} \mathrm{E}$ ), Klaipeda region (GPS coordinates: $55^{\circ} 36^{\prime} 1.66^{\prime \prime} \mathrm{N} 21^{\circ} 36^{\prime} 3.8^{\prime \prime} \mathrm{E} ; 55^{\circ} 34^{\prime} 50.6^{\prime \prime} \mathrm{N} 21^{\circ} 14^{\prime} 58.1^{\prime \prime} \mathrm{E}$ ) and Alytus region (GPS coordinates: $54^{\circ} 23^{\prime} 43.8^{\prime \prime} \mathrm{N}, 23^{\circ} 56^{\prime} 18.7^{\prime \prime} \mathrm{E}$ ) of Lithuania. Isolation and culturing methods have been described in detail by Stanevičienė et al. [16]. Generally, cultivable yeasts are isolated by direct rinsing of fruits with MD medium (2\% dextrose, 1\% (NH4)2SO4, $0.09 \% \mathrm{KH} 2 \mathrm{PO} 4,0.05 \% \mathrm{MgSO} 4,0.023 \% \mathrm{~K} 2 \mathrm{HPO} 4,0.01 \% \mathrm{NaCl}, 0.01 \% \mathrm{CaCl}_{2}$ ) or by applying fermentation-based enrichment. After cultivation on YPD-agar plates (1\% yeast extract, $1 \%$ peptone, $2 \%$ dextrose, $2 \%$ agar), morphologically distinct colonies proceeded molecular analysis. For taxonomic identification of the isolates, the ITS1-5.8S rDNA-ITS2 region or D1/D2 region of 26S rDNA were PCR-amplified as described in Stanevičienè et al. [16]. To assess their taxonomic position, the resolved sequences were compared with those available in the current version of the GenBank database at the National Centre for Biotechnology Information (NCBI) (Table A1).

\subsection{Insects}

European cherry fruit flies, Rhagoletis cerasi (L.) (Diptera: Tephritidae), were collected as pupae from soil under sweet and sour cherry trees in April of 2019-2021 at private

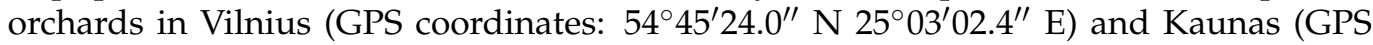
coordinates: $54^{\circ} 54^{\prime} 13.8^{\prime \prime} \mathrm{N} 23^{\circ} 48^{\prime} 07.9^{\prime \prime} \mathrm{E}$ ) districts, Lithuania. Reactivation of the pupae took place in a climate chamber "Fitotron" under 20-24 ${ }^{\circ} \mathrm{C}, 16 \mathrm{~L}: 8 \mathrm{D}$ (light:dark) photoperiod, and $65-75 \%$ relative humidity. Each pupa was placed in an individual $14 \mathrm{~mL}$ glass vial bearing wet $3 \mathrm{~cm}^{2}$ filter paper inside and closed by foam stoppers. The filter paper was humidified periodically to keep the humidity up inside the vial. After emergence, the adults were kept within the same vials in the room under $18-20{ }^{\circ} \mathrm{C}$, a natural daylight photoperiod, $50-60 \%$ relative humidity, and fed on $10 \%$ sugar solution in water. The flies possessing an ovipositor were attributed to females. After sexing, each individual was kept in a separate vial under the identical conditions as described above.

\subsection{Sampling and Analysis of Volatiles Produced by Yeasts}

For sampling of volatile organic compounds, yeasts were selected based on isolation frequency over the different ripening stages of cherries (Figure A1). The methods have been described in detail by Lukša et al. [7]. In summary, overnight grown yeast cells $(50 \mu L)$ at the concentration of about $3-5 \times 10^{7}$ cells $/ \mathrm{mL}$ were placed on the surface of YPD-agar medium and cultivated for two days at $25^{\circ} \mathrm{C}$. The solid-phase micro-extraction (SPME) technique was used to sample the headspace volatiles produced by yeasts. For sampling background volatiles, YPD-agar plates without yeast were used as control samples. The SPME needle was placed above the yeast culture through a small hole drilled in a Petri dish; the purified fibre coated with a polydimethylsiloxane-divinylbenzene absorbent $(65 \mathrm{~mm}$ coating layer thickness) was exposed to the headspace for $60 \mathrm{~min}$ at room temperature. The volatiles collected on the fibre were desorbed for $2 \mathrm{~min}$ in the injection liner of a gas chromatograph (GC).

GC and mass spectrometer (MS) were used to analyse the collected volatiles. The compounds were separated by a DB-Wax column under the subsequent temperature program: isothermal at $40^{\circ} \mathrm{C}$ for $1 \mathrm{~min}$ and afterwards gradually increased to $200{ }^{\circ} \mathrm{C}$ at a 
rate of $5^{\circ} \mathrm{C} / \mathrm{min}$, then to $240^{\circ} \mathrm{C}$ at a rate of $10^{\circ} \mathrm{C} / \mathrm{min}$, and maintained isothermally for $11 \mathrm{~min}$. The GC injector was run isothermally at $240{ }^{\circ} \mathrm{C}$. Helium served as a carrier gas. The relative amount of each of the compounds was determined based on the area of the chromatographic peak. The volatile compounds were identified by comparing their mass spectra and retention indexes with those presented in a NIST version 2.0 mass spectral library and those of the available synthetic standards. $C_{8}-C_{28} n$-alkanes were used to calculate the retention indexes of the volatiles.

\subsection{Gas Chromatography-Electroantennogram Detection}

Gas chromatographic and electroantennogram detection (EAD) techniques were applied to determine yeasts produced olfactory active volatiles to $R$. cerasi flies. A detailed description of the GC-EAD setup as well as the procedure has been published by Būda et al. [17]. Briefly, the GC was set up with a polar DB-Wax column. The injector and the detector were run at $240{ }^{\circ} \mathrm{C}$. The oven temperature was maintained at $40{ }^{\circ} \mathrm{C}$ for $1 \mathrm{~min}$; afterwards, it was raised to $240{ }^{\circ} \mathrm{C}$ at a rate of $10^{\circ} \mathrm{C} / \mathrm{min}$, then maintained isothermally for $13 \mathrm{~min}$. Hydrogen, at a flow rate of $1.5 \mathrm{~mL} / \mathrm{min}$, was used as a carrier gas. At the end of the GC column, a splitter divided an eluent into two equal parts, allowing simultaneous flame ionisation (FID) and EAD detection of the separated volatiles. A nitrogen make-up gas at $5 \mathrm{~mL} / \mathrm{min}$ flow rate was added to increase FID sensitivity. The part of an eluent allocated to EAD was mixed with charcoal filtered and humidified air flowing at $0.5 \mathrm{~m} / \mathrm{s}$ through a glass tube over antenna preparation. Glass capillary electrodes were used. The EAD and the FID signals were registered simultaneously, saved, and analysed. Before EAD recording, the antenna was stimulated with $1 \mu \mathrm{g}$ of 3-methyl-1-butanol to check sensitivity. Four to seven days old flies were used in the tests. Each antenna tested was from a different fly. In total, 21 antennae of males and 18 antennae of females were used.

\subsection{Electroantennogram Dose-Response}

The same electrophysiological recording setup and the antennal preparation technique were used to record electroantennogram (EAG) dose-responses of male and female flies to the synthetic EAD active compounds: 3-methylbutyl acetate; 3-methylbutyl propionate; 2-methyl-1-butanol; and 3-methyl-1-butanol.

The compounds were tested at the doses of $10^{-5}, 10^{-4}, 10^{-3}, 10^{-2}$, and $10^{-1} \mathrm{mg}$ applied in $10 \mu \mathrm{L}$ hexane on filter paper $(5 \times 45 \mathrm{~mm})$. The compounds were selected randomly, and five doses of each compound were tested in ascending order. A solvent blank (10 $\mu \mathrm{L}$ of hexane after evaporation) was tested as a control stimulus both at the beginning and the end of stimulation with each compound. Each EAD test was replicated 13 times, and each antenna used was from a different fly. The EAG response (R) to the EADactive compound dose was calculated according to the formula $\mathrm{R}=\mathrm{RA}-\left(\mathrm{RC}_{1}+\mathrm{RC}_{2}\right) / 2$, where RA is the EAG response to the EAD active compound, and RC1 and RC2 are EAG responses to the first and the second control stimuli, respectively.

\subsection{Behavioural Assay}

To test the behavioural choice of the flies to the synthetic EAD active compounds versus the control, a Y-tube olfactometer [18] $\left(25 \mathrm{~cm}\right.$ main tube, $17 \mathrm{~cm}$ arms, $110^{\circ}$ branching angle, the inner diameter of each arm and main tube $5 \mathrm{~cm}$ ) was used. The olfactometer was placed in a fume cupboard. Four T8/840, Colourlux plus, $18 \mathrm{~W}$ tube type lamps (NARVA Lichtquellen $\mathrm{GmbH}+\mathrm{Co}$. KG, Brand-Erbisdorf, Germany) covered with a white, mat, plastic shield (65 cm length, $42 \mathrm{~cm}$ width) at a distance of $23 \mathrm{~cm}$ were placed in front of the $\mathrm{Y}$ tube of the olfactometer. For the fruit flies, positive phototaxis is characteristic, and the light slightly stimulated the insects to move towards the light source. Each arm of the olfactometer was connected to a glass tube that contained either the stimulus or control. A purified air delivery system CADS-4CPP (Sigma Scientific LLC, Micanopy, FL, USA) was used to push air at a rate of $0.5 \mathrm{~L} / \mathrm{min}$ through each arm. 
The synthetic 3-methylbutyl propionate, 2-methyl-1-butanol, and 3-methyl-1-butanol were dissolved in hexane while paraffin oil was used to dissolve 3-methylbutyl acetate and the four component mixture. Behaviour modifying effect was assessed at the few doses by dispensing the $10 \mu \mathrm{L}$ of the solution on a filter paper strip $(5 \times 40 \mathrm{~mm})$. The proportion of EAD active components in the mixture used in the bioassay was based on the proportion of EAD active compounds determined in the sample of H. uvarum yeasts. The synthetic mixture consisted of 3-methylbutyl acetate $0.55 \mathrm{mg}$, 3-methylbutyl propionate $0.05 \mathrm{mg}$, 2-methyl-1-butanol $0.012 \mathrm{mg}$, and 3-methyl-1-butanol $0.28 \mathrm{mg}$ per $10 \mu \mathrm{L}$ of paraffin oil. After $0.5 \mathrm{~min}$ of solvent evaporation (only applicable for samples that were dissolved in hexane and no evaporation was carried out when paraffin oil was used), the filter paper strip was placed in the glass tube connected to one arm of the olfactometer. The same size filter paper was treated either with $10 \mu \mathrm{L}$ of hexane or with paraffin oil and was placed in the other arm serving as the control. After each test, the olfactometer was taken apart and the glassware was cleaned with hexane, soaked overnight in distilled water, and dried for $2 \mathrm{~h}$ in an oven, raising the temperature to $200{ }^{\circ} \mathrm{C}$. Silicone parts of the Y-tube olfactometer were cleaned with hexane, soaked overnight in distilled water, and air-dried or replaced between the tests.

A single fly was released into the $\mathrm{Y}$ olfactometer at the end of the main tube. The duration within which a fly must have reached the branch point was set to $15 \mathrm{~min}$. A fly was considered to have made a choice when it reached the distal end of the glass tube containing either a stimulus or a control (solvent after evaporation), irrespectively of whether the fly switched arms or not before reaching the odour source. The fly was considered as not making a choice if none of the arms was chosen within $15 \mathrm{~min}$. After every five tests, the positions of the two Y-tube arms were reversed. All insects were observed individually and used in a bioassay only once. The tests were carried out at $23 \pm 2{ }^{\circ} \mathrm{C}, 60 \% \mathrm{RH}$, between $10 \mathrm{~h}$ AM and $5 \mathrm{~h}$ PM local time.

\subsection{Statistical Analysis}

A nonparametric Mann-Whitney $U$ test was applied to evaluate differences in the volatile amounts between the yeast and control samples. To assess and visualise the associations between odour blends of eleven yeast species and volatile compounds, a multidimensional scaling (MDS) analysis with a Bray-Curtis index was performed on absolute amounts expressed as areas under chromatographic peaks using $\mathrm{R}$ (version 4.0.2) and Rstudio (version 1.3.959), with the metaMDS function in the vegan package (version 2.5-6), and the results were visualised using ggplot2 (version 3.3.2). Prior to analysis, the data were log-transformed. Dendrogram of the odour blends was obtained by cluster analysis based on Euclidean distance using the same way transformed data as in the MDS analysis. The clustering was carried out based on the average of quantified volatile compounds from three different isolates per species. The different clusters were identified by visually evaluating the clustering. Paired $t$ test was applied to compare EAG amplitudes of $R$. cerasi antennae of males versus females at each dose tested. To evaluate the choices of flies to the synthetic EAD active compounds versus the control, the total number of flies that made a choice was analysed with a $\chi 2$ test (observed vs. expected). All the analysis except MDS was performed using Statistica 6.0 software (StatSoft, Inc., Tulsa, OK, USA).

\section{Results}

\subsection{Composition of Yeast Produced Volatile Blends}

Analysis of yeast produced volatiles revealed 82 compounds that were exclusively present in the headspace of eleven yeast species (Table A1) or occurred at significantly larger amounts compared to those of the control samples. The esters represented by 41 compounds were accounted as the most abounded group of volatiles released by yeasts followed by 18 alcohols, nine compounds bearing aromatic moiety, eight ketones, six fatty acids, four terpenoids, three lactones, and one each of isothiocyanate, furane, and sulphide functional group was detected once (Table 1). Compounds bearing ester, alcohol, aromatic, 
ketone, and fatty acid moiety were detected in the volatile blends of all yeast species while terpenoid, lactone, isothiocyanoate, furane, and sulphide type volatiles were emitted by the yeast of single or few species (Figure 1).

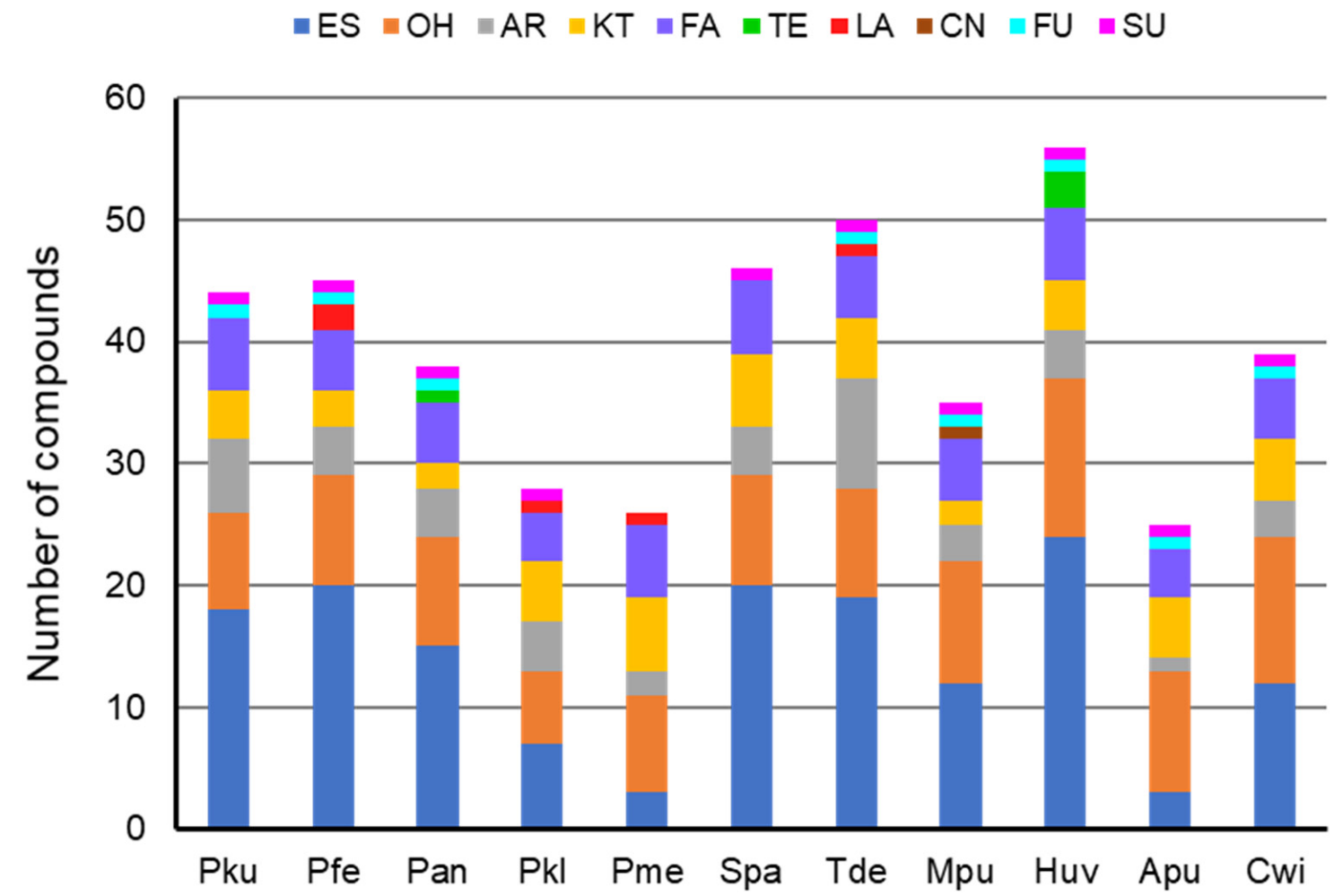

Figure 1. Chemical diversity of the volatile blends produced by yeasts. Pku-Pichia kudriavzevii, PfeP. fermentans, Pan-P. anomala, Pkl-P. kluyveri, Pme-P. membranifaciens, Spa-Saccharomyces paradoxus, TdeTorulaspora delbrueckii, Mpu-Metschnikowia pulcherrima, Huv-Hanseniaspora uvarum, Apu-Aureobasidium pullulans, Cwi-Cryptococcus wieringae. Functional group of volatiles: ES-ester; OH-alcohol; ARaromatic; KT-ketone; FA-fatty acid; TE-terpenoid; LA-lactone; CN-isothiocyanoate; FU-furane; SU-sulphide.

Nonmetric multidimensional scaling analysis showed that volatile blends of all eleven species grouped in a species-specific manner (Figure 2).

$P$. kudriavzevii and $H$. uvarum yeasts released the most similar volatile blends and together with $P$. anomala as well as $M$. pulcherrima yeasts formed a distinct cluster. Yeasts of P. fermentans, P. membranifaciens, A. pullulans, T. delbrueckii, and S. paradoxus clustered in another group. The most different blends were produced by P. kluyveri and C. wieringae yeasts (Figure 3).

\subsection{EAD Active Compounds}

GC-EAD analyses of the headspace collections from five yeast species representing three fruit ripening stages showed that antennae of $R$. cerasi flies, a pest of cherry fruits, responded to 3-methybutyl acetate, 3-methylbutyl propionate, 2-methyl-1-butanol, and 3-methyl-1-butanol (Figure 4, Table 2). Antennae of both R. cerasi sexes responded to all four EAD active compounds (Table 2). 


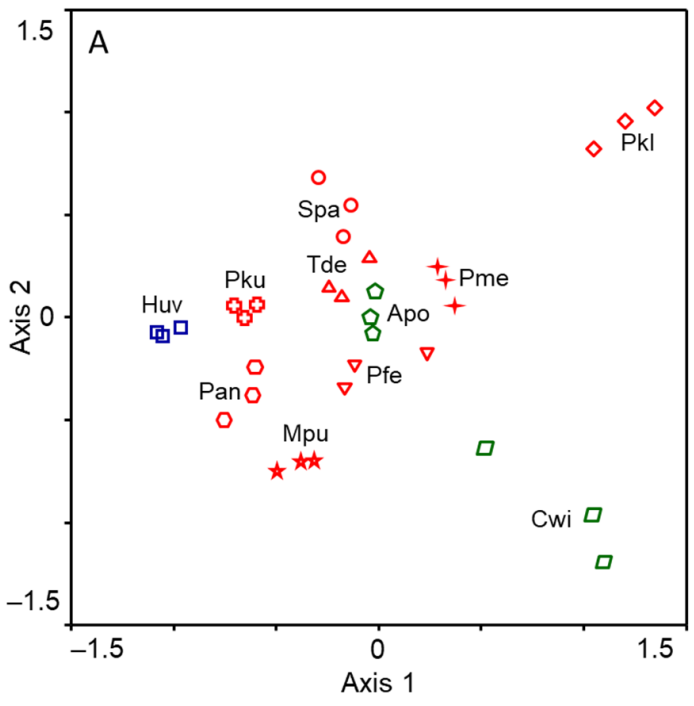

(a)

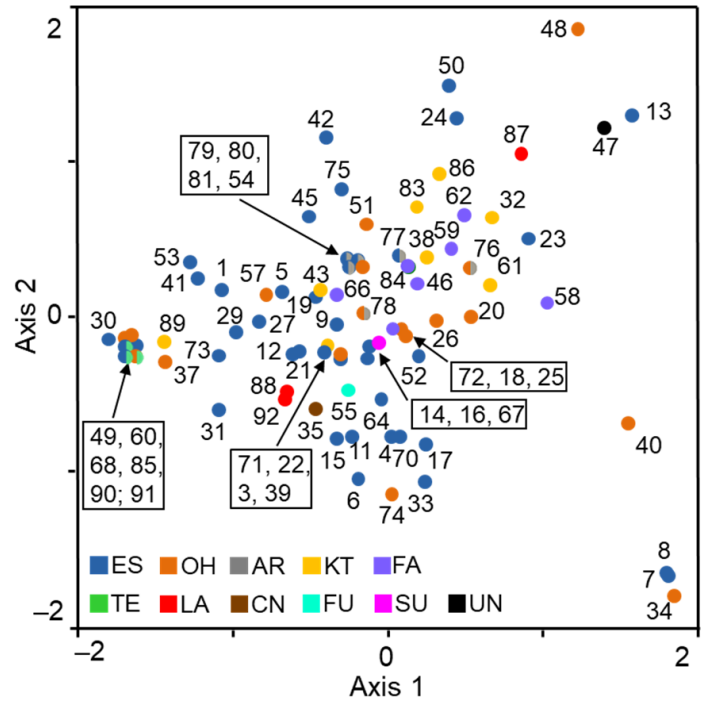

(b)

Figure 2. Multidimensional scaling plots: (a) eleven yeast species (each represented by three different isolates); (b) volatile compounds produced by yeasts. Volatiles were sampled from the headspace by SPME. Pku—Pichia kudriavzevii, Pfe-P. fermentans, Pan-P. anomala, Pkl—P. kluyveri, PmeP. membranifaciens, Spa-Saccharomyces paradoxus, Tde-Torulaspora delbrueckii, Mpu-Metschnikowia pulcherrima, Huv-Hanseniaspora uvarum, Apu-Aureobasidium pullulans, Cwi-Cryptococcus wieringae. $\mathrm{Apu}$ and Cwi yeasts are more common on unripe fruits and their symbols are coloured green, Huv yeasts are the most common on medium-ripe and ripe fruits and are indicated by the blue colour, and the red colour represents yeasts, the most common on ripe fruits. The name of volatiles indicated by numbers are listed in Table 1. Functional group of volatiles: ES-ester; $\mathrm{OH}$-alcohol; $\mathrm{AR}$-aromatic; KT—ketone; FA—fatty acid; TE—-terpenoid; LA—lactone; CN—isothiocyanoate; FU—furane; SU—sulphide; UN-unidentified.

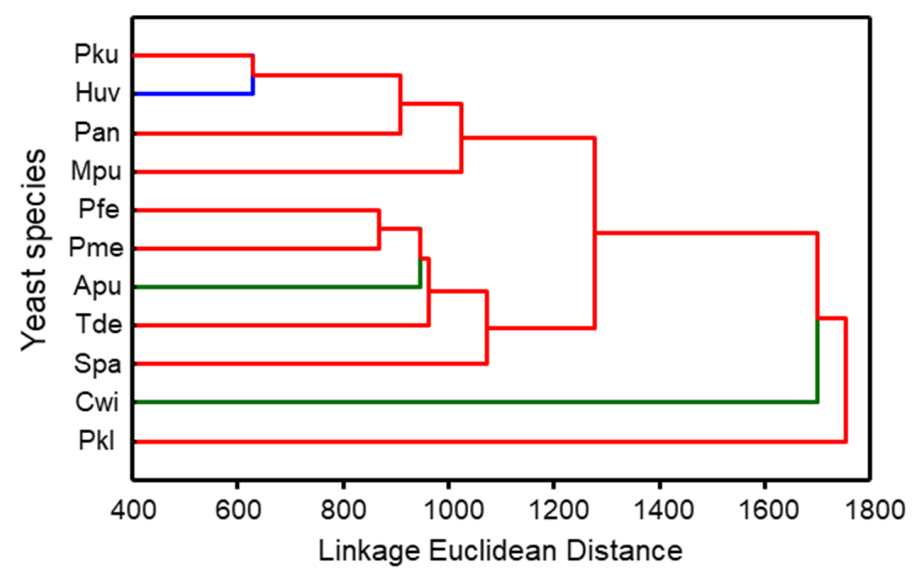

Figure 3. Dendrogram of odour blends sampled by SPME from the headspace of eleven yeast species. The dendrogram was obtained by cluster analysis based on Euclidean distance. The clustering was carried out based on the average of quantified volatile compounds from three different isolates per species. The different clusters were identified by visually evaluating the clustering. PkuPichia kudriavzevii, Pfe-P. fermentans, Pan-P. anomala, Pkl—P. kluyveri, Pme-P. membranifaciens, Spa-Saccharomyces paradoxus, Tde-Torulaspora delbrueckii, Mpu-Metschnikowia pulcherrima, HuvHanseniaspora uvarum, Apu-Aureobasidium pullulans, Cwi-Cryptococcus wieringae. Apu and Cwi yeasts are more common on unripe fruits and are represented by the green colour, Huv yeasts are the most common on medium-ripe and ripe fruits and are indicated by the blue colour, and the red colour represents yeasts, the most common on ripe fruits. 
Table 1. Odour blends of eleven yeast species and controls sampled from a headspace by the SPME technique.

\begin{tabular}{|c|c|c|c|c|c|c|c|c|c|c|c|c|c|c|c|c|}
\hline No & Compound & $\mathrm{CAS} \mathrm{No}^{3}$ & $\mathrm{RI}^{4}$ & $\mathrm{GR}^{5}$ & Control & A. pullulans & C. wieringae & H. иvarum & P. kudriavzevii & P. fermentans & P. anomala & P. kluyveri & P. membranifac & S. paradoxus & T. delbrueckii & M. pulcherrima \\
\hline 1 & Ethyl acetate & $141-78-6$ & $>900$ & $\mathrm{ES}^{6}$ & $11^{19} \pm 6$ & 0 & 0 & $2256 \pm 657$ & $2092 \pm 150$ & $1.6 \pm 1.2$ & $2816 \pm 116$ & 0 & 0 & $118 \pm 76$ & $202 \pm 55$ & $1586 \pm 765$ \\
\hline 2 & 3-Methylbutanal $* 1$ & $590-86-3$ & 927 & $\mathrm{AL}^{7}$ & $38 \pm 8$ & 0 & 0 & 0 & 0 & 0 & 0 & 0 & 0 & $15 \pm 8$ & 0 & 0 \\
\hline 3 & $\begin{array}{l}\text { Ethenol } \\
\text { Ethanol }\end{array}$ & $64-17-5$ & 944 & $\mathrm{OH}^{8}$ & $\begin{array}{c}0.16 \\
0\end{array}$ & $707 \pm 106$ & $48 \pm 11$ & $386 \pm 34$ & $425 \pm 5$ & $152 \pm 23$ & $39 \pm 23$ & 0 & 0 & $2594 \pm 1683$ & $468 \pm 19$ & $677 \pm 44$ \\
\hline 4 & Ethyl propionate & $1105-37-3$ & 953 & ES & 0 & 0 & $0.9 \pm 0.3$ & $139 \pm 38$ & $149 \pm 9$ & 0 & $147 \pm 14$ & 0 & 0 & $1.00 \pm 0.05$ & $63 \pm 8$ & $65 \pm 25$ \\
\hline 5 & Ethyl 2-methylpropionate & $97-62-1$ & 960 & ES & 0 & 0 & 0 & 0 & 0 & $0.9 \pm 0.7$ & 0 & 0 & 0 & & 0 & 0 \\
\hline $\begin{array}{l}6 \\
7\end{array}$ & $\begin{array}{l}\text { Propyl acetate } \\
\text { Methle -methlybutanoate }\end{array}$ & $\begin{array}{l}109-60-4 \\
866-57-5\end{array}$ & $\begin{array}{l}969 \\
989\end{array}$ & $\begin{array}{l}\text { ES } \\
\text { ES }\end{array}$ & ${ }_{0}^{0}$ & 0 & $\begin{array}{c}0 \\
08 \\
+002\end{array}$ & $8.0 \pm 0.7$ & $\begin{array}{l}0 \\
0\end{array}$ & 0 & $99 \pm 11$ & 0 & 0 & 0 & 0 & $6.3 \pm 3.6$ \\
\hline & $\begin{array}{l}\text { Methlyl 2-methlylubatanoate } \\
\text { Methyl 3-methylbutanoate }\end{array}$ & $\begin{array}{l}86-87-5 \\
556-24-1\end{array}$ & $\begin{array}{l}989 \\
996\end{array}$ & $\begin{array}{l}\text { ES } \\
\text { ES }\end{array}$ & $\begin{array}{l}0 \\
0\end{array}$ & $\begin{array}{l}0 \\
0\end{array}$ & $\begin{array}{l}0.8 \pm 0.2 \\
0.7 \pm 0.2\end{array}$ & $\begin{array}{l}0 \\
0\end{array}$ & $\begin{array}{l}0 \\
0\end{array}$ & $\begin{array}{l}0 \\
0\end{array}$ & $\begin{array}{l}0 \\
0\end{array}$ & $\begin{array}{l}0 \\
0\end{array}$ & $\begin{array}{l}0 \\
0\end{array}-P$ & $\begin{array}{r}0 \\
0\end{array}$ & $\begin{array}{l}0 \\
0\end{array}$ & $\begin{array}{l}0 \\
0\end{array}$ \\
\hline $\begin{array}{l}8 \\
9\end{array}$ & $\begin{array}{l}\text { Methly1-methlybutanaoate } \\
\text {-Methylprop-1-yl acetatate }\end{array}$ & $\begin{array}{l}55-24-1 \\
110-19-0\end{array}$ & $\begin{array}{l}996 \\
994\end{array}$ & $\begin{array}{l}\mathrm{ES} \\
\mathrm{ES} \\
\mathrm{ES}\end{array}$ & $\begin{array}{l}0 \\
0\end{array}$ & $\begin{array}{l}0 \\
0\end{array}$ & $\frac{0.7 \pm 0.2}{0}$ & $15 \pm 5$ & $91 \pm 6$ & $\begin{array}{l}0 \\
0\end{array}$ & $59 \pm 19$ & $\begin{array}{ll}0 \\
0.4 \pm 0.4\end{array}$ & $\begin{array}{l}0 \\
0\end{array}$ & $\begin{array}{l}0.0 \\
5.4 \pm 2.0\end{array}$ & $12 \pm 3$ & $8.9 \pm 2.1$ \\
\hline 10 & Toluene* & $108-88-3$ & 1013 & $\mathrm{AR}^{9}$ & $6.3 \pm 1.0$ & $1.9 \pm 0.1$ & $1.7 \pm 0.2$ & $1.3 \pm 0.6$ & $0.8 \pm 0.3$ & $2.2 \pm 0.3$ & $0.5 \pm 0.1$ & $1.8 \pm 0.8$ & $3.8 \pm 0.2$ & $3.8 \pm 1.7 \mathrm{~ns}$ & $2.2 \pm 0.7$ & $1.6 \pm 0.1$ \\
\hline 11 & Ethyl butanoate & $105-54-4$ & 1015 & ES & 0 & 0 & 0 & $0.8 \pm 0.5$ & $1.1 \pm 0.1$ & $0.5 \pm 0.4$ & $0.6 \pm 0.1$ & 0 & 0 & $8.8 \pm 0.8$ & $1.2 \pm 0.3$ & $0.5 \pm 0.4$ \\
\hline 12 & $\begin{array}{l}\text { Ethyl } 2 \text {-methylbutanoate } \\
\text { 3ethyth thl formote }\end{array}$ & 7452-79-1 & 1035 & ES & 0 & $3.6 \pm 0.5$ & $1.6 \pm 0.3$ & $1.3 \pm 0.5$ & $1.2 \pm 0.2$ & $4.3 \pm 3.7$ & $3.8 \pm 0.9$ & 0 & 0 & $6.6 \pm 0.2$ & $2.6 \pm 0.2$ & $3.5 \pm 0.5$ \\
\hline $\begin{array}{l}13 \\
14\end{array}$ & $\begin{array}{l}\text { 3-Methylbutyl formate } \\
\text { Ethyl 3-methylbutanoate }\end{array}$ & $\begin{array}{l}110-45-2 \\
108-6-2\end{array}$ & $\begin{array}{l}1053 \\
1053 \\
-1030\end{array}$ & $\begin{array}{l}\mathrm{ES} \\
\mathrm{ES}\end{array}$ & $\begin{array}{l}0 \\
0\end{array}$ & $\begin{array}{c}0 \\
0.4 \pm 0.1\end{array}$ & $\begin{array}{c}0 \\
0.10 \pm 0.02\end{array}$ & ${ }_{0}^{0}$ & $\begin{array}{l}0 \\
0\end{array}$ & $\begin{array}{c}0 \\
3.9 \pm 2.8\end{array}$ & 0 & $\frac{1.0 \pm 0.4}{0}$ & 0 & $\begin{array}{c}0 \\
17+04\end{array}$ & $\begin{array}{c}0 \\
07+01\end{array}$ & $\stackrel{0}{0}$ \\
\hline & $\begin{array}{l}\text { Ethyl 13-methylbutanoate } \\
\text { Butyl acetate }\end{array}$ & $\begin{array}{l}108-6-56-5 \\
12-86-4\end{array}$ & 1053 & $\begin{array}{l}\text { ES } \\
\text { ES }\end{array}$ & $\begin{array}{l}0 \\
0\end{array}$ & $0.4 \pm 0.1$ & $\begin{array}{l}0.10 \pm 0 \\
0\end{array}$ & $\begin{array}{c}0 \\
12 \pm 2\end{array}$ & $\begin{array}{c}0 \\
1.8 \pm 0.2\end{array}$ & $\begin{array}{l}3.9 \pm 2.8 \\
0\end{array}$ & $\begin{array}{c}0 \\
46 \pm 4\end{array}$ & $\begin{array}{l}0 \\
0\end{array}$ & $\begin{array}{l}0 \\
0\end{array}$ & $\begin{array}{l}1.7 \pm 0.4 \\
0\end{array}$ & $\begin{array}{l}0.7 \pm 0.1 \\
0.7 \pm 0.4\end{array}$ & $\begin{array}{l}0.6 \pm 0.2 \\
0.15+0.03\end{array}$ \\
\hline 16 & 2-Methylpropyl propionate & $\begin{array}{l}123-80-4 \\
540-42-1\end{array}$ & 1064 & $\begin{array}{l}\mathrm{ES} \\
\mathrm{ES}\end{array}$ & 0 & ${ }_{0}^{0}$ & $0.4 \pm 0.1$ & $\begin{array}{l}1 . \pm 2 \\
1.1 \pm 0.2\end{array}$ & $\begin{array}{l}1.8 \pm 0.2 \\
3.7 \pm 0.5\end{array}$ & $1.7 \pm 0.9$ & $\begin{array}{c}46 \pm 4 \\
3.6 \pm 0.8\end{array}$ & $496 \pm 496$ & 0 & $0.8 \pm 0.1$ & $\begin{array}{l}0.7 \pm 0.4 \\
3.5 \pm 0.4\end{array}$ & $\begin{array}{c}0.15 \pm 0.03 \\
0.4 \pm 0.2\end{array}$ \\
\hline 17 & 2-Methylpropyl 2-methylpropionate & $\begin{array}{l}07-45-8 \\
97-85-8\end{array}$ & 1074 & ES & 0 & 0 & $0.2 \pm 0.1$ & 00.2 & 0 & $0.3 \pm 0.1$ & 00.0 & 0 & 0 & 0 & 00 & 0.8 \\
\hline 18 & 2-Methylpropanol 1 & $\begin{array}{l}78-83-1 \\
7123-9-2\end{array}$ & 1093 & $\mathrm{OH}_{\mathrm{FS}}$ & $\stackrel{0}{0}$ & $114 \pm 6$ & $143 \pm 12$ & $\begin{array}{r}53 \pm 9 \\
658+88\end{array}$ & $61 \pm 7$ & $106 \pm 17$ & $25 \pm 1$ & $56 \pm 14$ & $26 \pm 03$ & $97 \pm 6$ & $56 \pm 06$ & $102 \pm 20$ \\
\hline $\begin{array}{l}19 \\
20\end{array}$ & $\begin{array}{l}\text { 3-Methylbutyl acetate } \\
\text { 1-Butanol* }\end{array}$ & $123-92-2$ & 1109 & ES & $5.4 \pm 0.2$ & 200 & $4.3 \pm 1.1 \mathrm{~ns}$ & $658 \pm 88$ & $1202 \pm 167$ & $\begin{array}{l}117 \pm 84 \\
04+04 \mathrm{ng}\end{array}$ & $1934 \pm 948$ & $70 \pm 50$ & 0 & $188 \pm 55$ & $623 \pm 252$ & $49 \pm 19$ \\
\hline $\begin{array}{l}20 \\
21\end{array}$ & 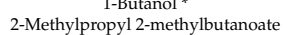 & $\begin{array}{l}71-36-3 \\
2445-67-2\end{array}$ & $\begin{array}{l}1144 \\
1166\end{array}$ & $\begin{array}{l}\mathrm{OH} \\
\mathrm{ES}\end{array}$ & $0.8 \pm 0.03$ & $2.9 \pm 0.6$ & ${ }_{0}^{18 \pm 5}$ & $\stackrel{4.4 \pm 0.6}{0}$ & $\frac{0.3 \pm 0.2}{0}$ & $\begin{array}{l}0.4 \pm 0.4 \mathrm{~ns} \\
0.7 \pm 0.1\end{array}$ & $\frac{1.6 \pm 0.6}{0}$ & $\begin{array}{l}0 \\
0\end{array}$ & $\begin{array}{l}0 \\
0\end{array}$ & $2.2 \pm 0.3$ & $\begin{array}{ll}0.4 \pm 0.2 \mathrm{~ns} \\
0\end{array}$ & $\begin{array}{l}4.5 \pm 1.0 \\
0\end{array}$ \\
\hline 22 & 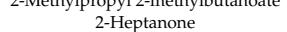 & $\begin{array}{l}2443-6-2 \\
110-43-0\end{array}$ & 1171 & $\begin{array}{l}\text { ES } \\
\text { KT } 10\end{array}$ & $\begin{array}{l}0 \\
0\end{array}$ & $\begin{array}{c}0 \\
10 \pm 8\end{array}$ & $\begin{array}{c}0 \\
8.0 \pm 1.7\end{array}$ & $\begin{array}{c}0 \\
0.5 \pm 0.1\end{array}$ & $\begin{array}{l}0 \\
0\end{array}$ & $\begin{array}{l}0.7 \pm 0.1 \\
2.6 \pm 1.1\end{array}$ & $\begin{array}{c}0 \\
0.2 \pm 0.04\end{array}$ & $1.9 \pm 0.9$ & $\begin{array}{c}0 \\
3.3 \pm 0.6\end{array}$ & $2.6 \pm 0.6$ & $4.8 \pm 0.6$ & $\begin{array}{c}0 \\
0.4 \pm 0.4\end{array}$ \\
\hline 23 & 3-Methylbutyl propionate & $105-68-0$ & 1178 & ES & $0.4 \pm 0.02$ & 0 & $0.3 \pm 0.2 \mathrm{~ns}$ & $\begin{array}{l}84 \pm 24 \\
84.1\end{array}$ & $23 \pm 4$ & $20 \pm 18$ & $71 \pm 8$ & $2937 \pm 1962$ & $1.6 \pm 0.7$ & $\begin{array}{l}2.010 .0 \\
11 \pm 1\end{array}$ & $\begin{array}{l}9.0 \pm 0.6 \\
98 \pm 11\end{array}$ & $1.4 \pm 0.5$ \\
\hline 24 & 3-Methylbuty 2 2-methylpropionate & $2050-01-3$ & 1184 & ES & 0 & 0 & 0 & 0 & 0 & $4.5 \pm 1.8$ & 0 & 0 & $0.5 \pm 0.2$ & $0.3 \pm 0.1$ & $12 \pm 3$ & 0 \\
\hline 25 & 2-Methyl-1-butanol & $137-32-6$ & 1205 & $\mathrm{OH}$ & 0 & $80 \pm 11$ & $262 \pm 64$ & $129 \pm 25$ & $201 \pm 61$ & $209 \pm 19$ & $96 \pm 11$ & $154 \pm 15$ & $29 \pm 4$ & $172 \pm 53$ & $199 \pm 37$ & $193 \pm 4$ \\
\hline${ }_{27}^{26}$ & $\begin{array}{l}\text { 3-Methyl-1-butanol } \\
\text { Ethyl hexanoate }\end{array}$ & $\begin{array}{l}123-5-1-3 \\
12-66-0\end{array}$ & $\begin{array}{l}1212 \\
1225\end{array}$ & $\begin{array}{c}\mathrm{OH} \\
\mathrm{ES}\end{array}$ & $0.8 \pm 0.1$ & $\begin{array}{l}640 \pm 86 \\
08+0.4\end{array}$ & $\begin{array}{c}889 \pm 297 \\
0\end{array}$ & $\begin{array}{l}461 \pm 84 \\
06\end{array}$ & $\begin{array}{l}874+74 \\
07+02\end{array}$ & $\begin{array}{l}1876 \pm 117 \\
3.6+36\end{array}$ & $\begin{array}{c}416 \pm 21 \\
0\end{array}$ & $\begin{array}{c}568 \pm 118 \\
0\end{array}$ & $687 \pm 0$ & $1418 \pm 93$ & $1787 \pm 61$ & $644 \pm 226$ \\
\hline 28 & $\begin{array}{l}\text { Ethyl hexanooate } \\
\text { Styrene* }\end{array}$ & $\begin{array}{l}12-306-0 \\
100-42-5\end{array}$ & 1237 & $\begin{array}{l}\mathrm{ES} \\
\mathrm{AR}\end{array}$ & $11 \pm 4$ & $\begin{array}{l}0.8 \pm 0.4 \\
2.4 \pm 2.0\end{array}$ & $19 \pm 5$ ns & $\begin{array}{l}0.0 \pm 0.1 \\
0.5 \pm 0.2\end{array}$ & $\begin{array}{r}0.7 \pm 0.2 \\
3.5 \pm 2.9 \mathrm{~ns} 20\end{array}$ & $\begin{array}{l}3.2 \pm 3.6 \\
5.2 \pm \mathrm{ns}\end{array}$ & $19 \pm 24 \mathrm{~ns}$ & $14 \pm 0.7 \mathrm{~ns}$ & $7.2 \pm 2.1 \mathrm{~ns}$ & $\begin{array}{l}16 \pm 9 \\
9 \pm 2 \mathrm{~ns}\end{array}$ & $\begin{array}{l}0.1 \pm 0.03 \\
10 \pm 3 \mathrm{~ns}\end{array}$ & $11 \pm 6 \mathrm{~ns}$ \\
\hline 29 & 3-Methylbutyl butanoate & $106-27-4$ & 1256 & ES & 0 & 0 & 0 & $0.4 \pm 0.1$ & 0 & $0.5 \pm 0.02$ & 0 & 0 & 0 & 0 & $0.4 \pm 0.1$ & 0 \\
\hline 31 & 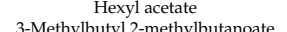 & $\begin{array}{l}142-92-7 \\
2762-35-0\end{array}$ & 1265 & $\begin{array}{l}\mathrm{ES} \\
\mathrm{FS}\end{array}$ & $\begin{array}{l}0 \\
0\end{array}$ & $\begin{array}{l}0 \\
0\end{array}$ & $\begin{array}{l}0 \\
0 \\
0\end{array}$ & $0.45 \pm 0.03$ & $\begin{array}{l}0 \\
0\end{array}$ & $\begin{array}{c}0 \\
30+08\end{array}$ & $\begin{array}{c}0 \\
15+03\end{array}$ & $0_{0}^{0}$ & ${ }_{0}^{0}$ & ${ }_{0}^{0}$ & $\begin{array}{l}0 \\
0\end{array}$ & ${ }_{0}^{0}$ \\
\hline $\begin{array}{l}31 \\
32\end{array}$ & $\begin{array}{l}\text { 3-Methylbuty I-methylyutanoate } \\
\text { 2-hydroxy-3-butanone }\end{array}$ & $\begin{array}{l}27625-85-0 \\
513-86-0\end{array}$ & $\begin{array}{l}1272 \\
1274\end{array}$ & $\begin{array}{l}\text { ES } \\
\text { KT }\end{array}$ & $\begin{array}{l}0 \\
0\end{array}$ & $2.2 \pm 0.9$ & $\begin{array}{c}0 \\
3.6 \pm 0.4\end{array}$ & $10 \pm 1$ & $\begin{array}{l}0 \\
0\end{array}$ & $\begin{array}{l}3.0 \pm 0.8 \\
0\end{array}$ & $1.5 \pm 0.3$ & $\begin{array}{c}0 \\
0.7 \pm 0.1\end{array}$ & $\begin{array}{l}0 \\
0\end{array}$ & $\begin{array}{l}0 \\
67+07\end{array}$ & $\begin{array}{c}0 \\
22+02\end{array}$ & $\begin{array}{c}0 \\
17+2\end{array}$ \\
\hline 33 & 3-Methylbutyly-3-methylbutuanoate & $\begin{array}{l}510-00-0 \\
659-70-1\end{array}$ & 1288 & $\begin{array}{l}\mathrm{N} \\
\mathrm{ES}\end{array}$ & 0 & $\frac{2.210 .9}{0}$ & $\begin{array}{l}3.0 \pm 0.4 \\
0.4 \pm 0.2\end{array}$ & ${ }_{0}^{10+1}$ & 0 & $8.9 \pm 3.9$ & $0.7 \pm 0.1$ & 0 & 0 & 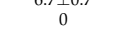 & $\frac{2.2 \pm 0.2}{0}$ & 0 \\
\hline 34 & 2-Methylpentanol & $105-30-6$ & 1299 & $\mathrm{OH}$ & 0 & 0 & $13 \pm 3$ & 0 & 0 & 0 & 0 & 0 & 0 & 0 & 0 & 0 \\
\hline 35 & 2-Methylpropyl isothiocyanoate & $591-82-2$ & 1304 & $\mathrm{CN}^{11}$ & 0 & 0 & 0 & 0 & 0 & 0 & 0 & 0 & 0 & 0 & 0 & $0.4 \pm 0.1$ \\
\hline 36 & 2,5- Dimethyl pyrazine * & $123-32-0$ & 1314 & $\mathrm{PY}^{12}$ & $9.1 \pm 0.5$ & $1.2 \pm 0.2$ & $22 \pm 13$ ns & $3.8 \pm 0.6$ & $3.4 \pm 0.4$ & $4.8 \pm 0.2$ & $2.0 \pm 0.6$ & $6.0 \pm 3.1 \mathrm{~ns}$ & $7.6 \pm 0.4 \mathrm{~ns}$ & $6.4 \pm 0.5 \mathrm{~ns}$ & $3.9 \pm 0.5$ & $4.6 \pm 0.6$ \\
\hline 37 & 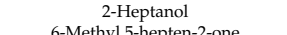 & $543-49-7$ & 1321 & $\underset{\mathrm{KT}}{\mathrm{OH}}$ & 0 & $2.0 \pm 1.3$ & ${ }_{103}^{0}$ & $0.50 \pm 0.01$ & 0 & 0 & 0 & 0 & 0 & 0 & 0 & $0.9 \pm 0.7$ \\
\hline 38 & $\begin{array}{l}\text { 6-Methyl 5-hepten-2-one } \\
\text { Ethyl heptanoate }\end{array}$ & $\begin{array}{l}110-93-0 \\
106-20-9\end{array}$ & ${ }_{1327}^{1327}$ & $\begin{array}{l}\mathrm{KT} \\
\mathrm{FS}\end{array}$ & $0.4 \pm 0.1$ & $4.4 \pm 3.8$ & $103 \pm 23$ & $0.29 \pm 0.03 \mathrm{~ns}$ & $\begin{array}{c}0 \\
04+01\end{array}$ & $0.7 \pm 0.1$ & $0_{0}^{0}-x-10$ & $0.8 \pm 0.5 \mathrm{~ns}$ & $2.8 \pm 1.0$ & $1.1 \pm 0.2$ & $0.5 \pm 0.4 \mathrm{~ns}$ & 0 \\
\hline $\begin{array}{l}39 \\
40\end{array}$ & $\begin{array}{l}\text { Ethyl heptanoate } \\
\text { 1-Hexanol }\end{array}$ & $\begin{array}{l}106-30-9 \\
111-27-3\end{array}$ & $\begin{array}{l}1331 \\
1346\end{array}$ & $\begin{array}{l}\mathrm{ES} \\
\mathrm{OH}\end{array}$ & $\begin{array}{l}0 \\
0\end{array}$ & $\begin{array}{l}0 \\
0\end{array}$ & $\begin{array}{c}0 \\
05+02\end{array}$ & $\begin{array}{l}0 \\
0\end{array}$ & $0.4 \pm 0.1$ & ${ }_{0}^{0}$ & ${ }_{0}^{0}$ & ${ }_{0}^{0}$ & $43+$ & ${ }_{0}^{0}$ & ${ }_{0}^{0}$ & $\begin{array}{l}0 \\
0\end{array}$ \\
\hline 41 & $\begin{array}{l}\text { 1-nexanor } \\
\text { Heptyl acetate }\end{array}$ & 112-06-1 & $\begin{array}{l}1340 \\
1369\end{array}$ & $\mathrm{ES}$ & 0 & 0 & $\frac{0.5}{0.2}$ & $0.7 \pm 0.1$ & 0 & 0 & 0 & 0 & . & $0.3 \pm 0.2$ & 0 & 0 \\
\hline 42 & 2-Ethylhexyl acetate & $103-09-3$ & 1382 & ES & 0 & 0 & 0 & 0 & 0 & 0 & 0 & 0 & 0 & $1.0 \pm 0.4$ & 0 & 0 \\
\hline & Nonan-2-one & $821-55-6$ & 1383 & KT & 0 & $49 \pm 28$ & $13 \pm 4$ & $0.5 \pm 0.2$ & $0.3 \pm 0.1$ & $2.3 \pm 1.0$ & $1.7 \pm 0.4$ & $1.3 \pm 0.2$ & $2.9 \pm 1.0$ & $5.2 \pm 4.2$ & $2.8 \pm 0.6$ & 0 \\
\hline${ }_{45}^{44}$ & $\begin{array}{l}\text { 2,3,5-T-Timethyl pyrazine * } \\
\text { Ethyl octanoate }\end{array}$ & $\begin{array}{l}14667-55-1 \\
106-32-1\end{array}$ & $\begin{array}{l}1397 \\
1427\end{array}$ & $\begin{array}{l}\mathrm{PY} \\
\mathrm{ES}\end{array}$ & $0.6 \pm 0.1$ & $0.6 \pm 0.1 \mathrm{~ns}$ & $\begin{array}{l}0.8 \pm 0.1 \mathrm{~ns} \\
0\end{array}$ & $\begin{array}{l}0.58 \pm 0.01 \mathrm{~ns} \\
4.1 \pm 0.1\end{array}$ & $\begin{array}{l}0.6 \pm 0.04 \mathrm{~ns} \\
3.7 \pm 0.6\end{array}$ & $\begin{array}{l}0.6 \pm 0.1 \mathrm{~ns} \\
0.2 \pm 0.2\end{array}$ & $\begin{array}{c}0.6 \pm 0.1 \mathrm{~ns} \\
0\end{array}$ & $\begin{array}{l}0.4 \pm 0.2 \mathrm{~ns} \\
0\end{array}$ & $\begin{array}{l}0.7 \pm 0.2 \mathrm{~ns} \\
0\end{array}$ & $\begin{array}{l}0.8 \pm 0.1 \mathrm{~ns} \\
56 \pm 5.2\end{array}$ & $0.5 \pm 0.4 \mathrm{~ns}$ & $0.60 \pm 0.02 \mathrm{~ns}$ \\
\hline $\begin{array}{l}43 \\
46\end{array}$ & $\begin{array}{l}\text { Ethyly octanoate } \\
\text { Acetic acid }\end{array}$ & $\begin{array}{l}10-25-1 \\
64-19-7\end{array}$ & $\begin{array}{l}1427 \\
1439\end{array}$ & $\mathrm{FA}^{13}$ & $\begin{array}{c}0 \\
0.3 \pm 0.01\end{array}$ & $\begin{array}{c}0 \\
0.7 \pm 0.01\end{array}$ & $4.5 \pm 3.5 \mathrm{~ns}$ & $\begin{array}{l}4.1 \pm 0.1 \\
69 \pm 11\end{array}$ & $\begin{array}{l}3.3 \pm 0.6 \\
5.3 \pm 3.9 \mathrm{~ns}\end{array}$ & $\begin{array}{l}0.2 \pm 0.2 \\
0.6 \pm 0.1\end{array}$ & $7.9 \pm 3.0$ & 0 & $0.8 \pm 0.1$ & $\begin{array}{l}5.7 \pm 5.2 \\
0.79 \pm 0.04\end{array}$ & $0.77 \pm 0.04$ & $1.1 \pm 0.4$ \\
\hline 47 & Unknown & & 1440 & & 0 & 0 & 0 & 0 & 0 & 0 & 0 & $10 \pm 4$ & 0 & 0 & 0 & 0 \\
\hline $\begin{array}{l}48 \\
49\end{array}$ & $\begin{array}{l}\text { 1-Octen-3-ol } \\
\text { 1-Heptanol }\end{array}$ & $\begin{array}{l}3391-86-4 \\
11-70-6\end{array}$ & $\begin{array}{l}1450 \\
1454\end{array}$ & $\begin{array}{l}\mathrm{OH} \\
\mathrm{OH}\end{array}$ & $\begin{array}{l}0 \\
0\end{array}$ & $\begin{array}{l}0 \\
0\end{array}$ & $\begin{array}{l}0 \\
0\end{array}$ & $\begin{array}{c}0 \\
0.8 \pm 0.2\end{array}$ & $\begin{array}{l}0 \\
0\end{array}$ & 0 & 0 & ${ }_{0}^{0}$ & $0.12 \pm 0.01$ & ${ }_{0}^{0}$ & 0 & ${ }_{0}^{0}$ \\
\hline $\begin{array}{l}49 \\
50\end{array}$ & $\begin{array}{l}\text { 1-Heptanol } \\
\text { 3-Methylbutyl hexanoate }\end{array}$ & $\begin{array}{l}111-7-7-6 \\
2198-61-0\end{array}$ & $\begin{array}{l}1454 \\
1457\end{array}$ & $\begin{array}{c}\mathrm{OH} \\
\mathrm{ES}\end{array}$ & $\begin{array}{l}0 \\
0\end{array}$ & $\begin{array}{l}0 \\
0\end{array}$ & $\begin{array}{l}0 \\
0\end{array}$ & $0.8 \pm 0.2$ & $\begin{array}{l}0 \\
0\end{array}$ & $\begin{array}{l}0 \\
0\end{array}$ & $\begin{array}{l}0 \\
0\end{array}$ & $\begin{array}{l}0 \\
0\end{array}$ & 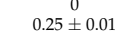 & $0.8 \pm 0.2$ & $\begin{array}{l}0 \\
0\end{array}$ & $\begin{array}{l}0 \\
0\end{array}$ \\
\hline 51 & 6-Methyl-5-hepten-2-ol & $1569-60-4$ & $\begin{array}{l}1457 \\
1460\end{array}$ & $\mathrm{OH}$ & 0 & $1.1 \pm 0.7$ & $12 \pm 3$ & 0 & 0 & 0 & 0 & 0 & $\begin{array}{l}0.20 \\
0\end{array}$ & $\underset{0}{0.01 .2}$ & 0 & 0 \\
\hline 52 & MHMP $^{2}$ & $40348-72-9$ & 1462 & $\mathrm{ES}$ & 0 & 0 & 0 & 0 & 0 & 0 & 0 & 0 & 0 & 0 & $1.2 \pm 0.3$ & 0 \\
\hline 53 & Octyl acetate & $112-14-1$ & 1472 & ES & 0 & 0 & 0 & $0.3 \pm 0.1$ & 0 & 0 & 0 & 0 & 0 & $1.3 \pm 0.9$ & 0 & 0 \\
\hline 54 & 2-Ethylhexanol & $104-76-7$ & 1483 & $\mathrm{OH}$ & $0.3 \pm 0.03$ & $1.5 \pm 0.1$ & $1.8 \pm 0.2$ & $0.8 \pm 0.1$ & $0.6 \pm 0.1$ & $0.6 \pm 0.1$ & $1.3 \pm 0.4$ & $0.8 \pm 0.4 \mathrm{~ns}$ & $1.7 \pm$ & $0.9 \pm 0.1$ & $1.1 \pm 0.2$ & $0.71 \pm 0.03$ \\
\hline 55 & Acetylfuran & $1192-62-7$ & 1496 & $\mathrm{FU}^{14}$ & 0 & $0.3 \pm 0.1$ & $0.3 \pm 0.2$ & $0.6 \pm 0.1$ & $0.2 \pm 0.1$ & $0.6 \pm 0.3$ & $0.7 \pm 0.3$ & 0 & 0 & 0 & $0.5 \pm 0.1$ & $0.19 \pm 0.02$ \\
\hline 56 & Benzaldehyde * & $100-52-7$ & 1505 & $\mathrm{AR}, \mathrm{AL}$ & $9.9 \pm 2.7$ & $2.0 \pm 1.6$ & $0.4 \pm 0.1$ & $0.4 \pm 0.1$ & $0.28 \pm 0.04$ & $0.29 \pm 0.04$ & $0.18 \pm 0.02$ & $1.0 \pm 0.2$ & $0.20 \pm 0.02$ & $0.23 \pm 0.01$ & $0.20 \pm 0.03$ & $0.3 \pm 0.1$ \\
\hline $\begin{array}{l}57 \\
58\end{array}$ & $\begin{array}{c}\text { 2-Nonanol } \\
\text { Propionic acid }\end{array}$ & $\begin{array}{l}628-99-9 \\
79-09-4\end{array}$ & $\begin{array}{l}1524 \\
1526\end{array}$ & ${ }_{\mathrm{FA}}^{\mathrm{OH}}$ & $\begin{array}{c}0 \\
0.3+0.04\end{array}$ & $\frac{0.05 \pm 0.04}{0}$ & $\begin{array}{c}0 \\
29+20 \mathrm{~ns}\end{array}$ & $\begin{array}{l}1.1 \pm \pm 0.2 \\
67+17\end{array}$ & $\begin{array}{c}0 \\
22 \\
+01\end{array}$ & $\begin{array}{l}0.2 \pm 0.1 \\
07+0.1 \mathrm{~ns}\end{array}$ & $\begin{array}{l}0 \\
0 \\
\end{array}$ & $\begin{array}{c}0 \\
0.9+0.2\end{array}$ & $\begin{array}{c}0 \\
16+03\end{array}$ & $0.3 \pm 0.1$ & $0.4 \pm 0.1$ & $0.5 \pm 0.1$ \\
\hline 59 & $\begin{array}{l}\text { Propionciacia } \\
\text { 2-Methylpropionic acid }\end{array}$ & $79-31-2$ & 1557 & FA & $\begin{array}{l}4.9 \pm 0.5 \\
4.9 \pm 0.5\end{array}$ & 0 & $12 \pm 6 \mathrm{~ns}$ & $34 \pm 5$ & $\begin{array}{l}2.220 .1 \\
26 \pm 2\end{array}$ & $\begin{array}{l}0.7 \pm 0.5 \mathrm{~ns} \\
13 \pm 7\end{array}$ & $\begin{array}{l}1.0 \pm 0.4 \\
9.9+2.2\end{array}$ & $\begin{array}{l}0.9 \pm 0.2 \\
8.3 \pm 1.6\end{array}$ & $\begin{array}{l}1.6 \pm 0.3 \\
20 \pm 3\end{array}$ & $\begin{array}{l}2.3 \pm 0.4 \\
18+12 \mathrm{ss}\end{array}$ & $\begin{array}{l}2.4 \pm 0.5 \\
19+08 n s\end{array}$ & $\begin{array}{l}0.3 \pm 0.1 \mathrm{~ns} \\
19+1.0 \mathrm{~ns}\end{array}$ \\
\hline 60 & 2-Decanol & $1120-06-5$ & 1574 & $\mathrm{OH}$ & 0 & 0 & 0 & $0.7 \pm 0.2$ & ${ }_{0}^{20 \pm 2}$ & $\begin{array}{c}13 \pm 7 \\
0\end{array}$ & $\begin{array}{l}9.9 \pm 2.2 \\
0\end{array}$ & $\begin{array}{l}8.3 \pm 1.6 \\
0\end{array}$ & 0 & $\begin{array}{l}1.8 \pm 1.2 \mathrm{~ns} \\
0\end{array}$ & $1.9 \pm 0.8 \mathrm{~ns}$ & $1.9=0$ \\
\hline 61 & 2-Undecanone & $112-12-9$ & 1594 & КТ & 0 & $1.1 \pm 0.6$ & $0.6 \pm 0.1$ & 0 & 0 & & 0 & $0.2 \pm 0.1$ & $1.1 \pm 0.2$ & $0.20 \pm 0.02$ & $0.8 \pm 0.2$ & \\
\hline 62 & Butanoic acid & $107-92-6$ & 1614 & FA & $1.0 \pm 0.1$ & $0.2 \pm 0.04$ & $2.6 \pm 1.8 \mathrm{~ns}$ & $8.5 \pm 1.7$ & $2.7 \pm 0.1$ & $0.7 \pm 0.5 \mathrm{~ns}$ & $9.0 \pm 2.1$ & $0.3 \pm 0.2$ & $1.1 \pm 0.1 \mathrm{~ns}$ & $0.2 \pm 0.1$ & $0.21 \pm 0.04$ & $0.3 \pm 0.2$ \\
\hline $\begin{array}{l}63 \\
64\end{array}$ & & $\begin{array}{l}98-86-2 \\
110-38-3 \\
\end{array}$ & $\begin{array}{l}1625 \\
1632\end{array}$ & $\begin{array}{l}\mathrm{AR}, \mathrm{KT} \\
\mathrm{ES}\end{array}$ & $1.8 \pm 0.2$ & $0.7 \pm 0.1$ & $\begin{array}{l}0.7 \pm 0.1 \\
0\end{array}$ & $\begin{array}{l}1.1 \pm 0.1 \\
40 \pm 12\end{array}$ & $0.8 \pm 0.04$ & $0.4 \pm 0.4$ & $0.9 \pm 0.1$ & $1.1 \pm 0.8 \mathrm{~ns}$ & $1.3 \pm 0.1 \mathrm{~ns}$ & $1.6 \pm 1.0 \mathrm{~ns}$ & $1.5 \pm 0.1 \mathrm{~ns}$ & $0.6 \pm 0.1$ \\
\hline $\begin{array}{l}84 \\
65\end{array}$ & $\begin{array}{l}\text { Ethyld decanoate } \\
\text { 2-Furanmethanol* }\end{array}$ & $\begin{array}{l}110-383-3 \\
98-00-0\end{array}$ & $\begin{array}{l}1032 \\
1649\end{array}$ & $\begin{array}{l}\mathrm{ES} \\
\mathrm{OH}\end{array}$ & $1.8 \pm 0.1$ & $1.7 \pm 1.5 \mathrm{~ns}$ & $1.2 \pm 0.4 \mathrm{~ns}$ & $\begin{array}{l}4.0 \pm 1.2 \\
0\end{array}$ & $0.9 \pm 0.1$ & $0.5 \pm 0.5$ & $\begin{array}{l}0.2 \pm 0.1 \\
0\end{array}$ & $\begin{array}{l}0 \\
0\end{array}$ & $\begin{array}{l}0 \\
0\end{array}$ & $\begin{array}{l}2.3 \pm 2.2 \\
0\end{array}$ & $\begin{array}{c}0 \\
1.7 \pm 0.4\end{array}$ & $\begin{array}{l}0 \\
0\end{array}$ \\
\hline
\end{tabular}


Table 1. Cont.

\begin{tabular}{|c|c|c|c|c|c|c|c|c|c|c|c|c|c|c|c|c|}
\hline No & Compound & $\mathrm{CASNo}^{3}$ & $\mathrm{RI}^{4}$ & $\mathrm{GR}^{5}$ & Control & A. pullulans & c. wieringae & H. uvarum & P. kudriavzevii & P. fermentans & P. anomala & P. kluyveri & P. membranifac & s. paradoxus & T. delbrueckii & M. pulcherrima \\
\hline 66 & 3-Methylbutanoic acid & $503-74-2$ & 1658 & FA & $20 \pm 2$ & $0.26 \pm 0.02$ & $58 \pm 39$ & $261 \pm 66$ & $136 \pm 9$ & $37 \pm 25$ ns & $44 \pm 12$ & $21 \pm 6 \mathrm{~ns}$ & $71 \pm 14$ & $6.8 \pm 5.6$ & $4.5 \pm 2.9$ & $4.6 \pm 4.1$ \\
\hline 67 & 3-Hydroxypropyl methylsulphide & $505-10-2$ & 1700 & su 15 & 0 & $0.18 \pm 0.03$ & $0.4 \pm 0.1$ & $0.33 \pm 0.04$ & $2.0 \pm 1.0$ & $1.3 \pm 0.7$ & $6.6 \pm 1.1$ & $0.9 \pm 0.4$ & 0 & $0.8 \pm 0.2$ & $7.5 \pm 2.2$ & $0.5 \pm 0.2$ \\
\hline 68 & Geranyl acetate & 16409-44-2 & 1764 & $\mathrm{TE}^{16}, \mathrm{ES}$ & 0 & 0 & 0 & $0.7 \pm 0.2$ & 0 & 0 & 0 & 0 & 0 & 0 & 0 & 0 \\
\hline 69 & Methoxy-phenyl-oxime * & $67160-14-9$ & 1768 & IM 17 & $3.1 \pm 0.7$ & $1.2 \pm 0.2$ & $2.9 \pm 0.4 \mathrm{~ns}$ & $0.6 \pm 0.3$ & $1.3 \pm 0.1$ & $0.7 \pm 0.2$ & $0.43 \pm 0.02$ & $1.7 \pm 0.4 \mathrm{~ns}$ & $1.0 \pm 0.3$ & $0.6 \pm 0.2$ & $0.6 \pm 0.2$ & $1.4 \pm 0.4$ \\
\hline 70 & & $119-43-7$ & 1767 & $\begin{array}{ll}\mathrm{AR}, \mathrm{ES} \\
\mathrm{AB}\end{array}$ & 0 & 0 & 0 & 0 & $0.6 \pm 0.2$ & 0 & $0.3 \pm 0.1$ & 0 & 0 & 0 & ${ }^{0}$ & 0 \\
\hline 71 & $\begin{array}{l}\text { 2-Phenylethyl acetate } \\
\text { Hexanoic acid }\end{array}$ & $\begin{array}{l}103-45-7 \\
122-70-3 \\
-1\end{array}$ & $\begin{array}{l}1794 \\
1816\end{array}$ & $\begin{array}{l}\text { AR, ES } \\
A R, E S\end{array}$ & $\begin{array}{l}0 \\
0 \\
0\end{array}$ & $\begin{array}{l}0 \\
0\end{array}$ & $\begin{array}{c}0 \\
0.8 \pm 0.1\end{array}$ & $148 \pm 13$ & $353 \pm 48$ & $\begin{array}{l}2.1 \pm 1.6 \\
0.3 \pm 0.2\end{array}$ & $88 \pm 8$ & $7.8 \pm 6.3$ & $\begin{array}{l}0 \\
0\end{array}$ & $8.0 \pm 3.4$ & $65 \pm 17$ & $11 \pm 8$ \\
\hline 73 & $\begin{array}{l}\text { Hexanoic acid } \\
\text { Ethyl dodecanoate }\end{array}$ & $\begin{array}{l}127-70-3 \\
106-33-2\end{array}$ & 1823 & AR, ES & $\begin{array}{l}0 \\
0 \\
0\end{array}$ & $\begin{array}{l}0 \\
0 \\
0\end{array}$ & $0.8 \pm 0.1$ & $1.7 \pm 1.0$ & $\begin{array}{l}0.4 \pm 0.1 \\
0.1 \pm 0.03\end{array}$ & $\begin{array}{l}0.3 \pm 0.2 \\
0.3 \pm 0.1\end{array}$ & $\begin{array}{l}0 \\
0\end{array}$ & $\begin{array}{l}0 \\
0\end{array}$ & $\begin{array}{l}0 \\
0 \\
0\end{array}$ & $0.6 \pm 0.1$ & ${ }_{0}^{0}$ & $\begin{array}{l}0 \\
0\end{array}$ \\
\hline 74 & Geraniol & $106-24-1$ & 1842 & TEOH & 0 & 0 & $0.5 \pm 0.1$ & 0 & 0 & 0 & $0.5 \pm 0.1$ & 0 & 0 & 0 & 0 & 0 \\
\hline 75 & 3-Methylbutyl decanoate & $2306-91-4$ & 1843 & ES & 0 & 0 & 0 & 0 & 0 & 0 & 0 & 0 & 0 & $0.2 \pm 0.1$ & 0 & 0 \\
\hline $\begin{array}{l}76 \\
77\end{array}$ & $\begin{array}{l}\text { Phenyl methanol } \\
\text { 2-Phennlethyl propionate }\end{array}$ & $\begin{array}{l}100-51-6 \\
127-70-3 \\
-10\end{array}$ & $\begin{array}{l}1854 \\
1862\end{array}$ & $\mathrm{AR}, \mathrm{OH}$ & $0.7 \pm 0.04$ & 0 & $0.7 \pm 0.2 \mathrm{~ns}$ & $\begin{array}{c}5.1 \pm 0.9 \\
18+4\end{array}$ & $2.9 \pm 0.5$ & $1.8 \pm 1.0 \mathrm{~ns}$ & $1.2 \pm 0.3 \mathrm{~ns}$ & $1.2 \pm 0.2$ & $0.6 \pm 0.2 \mathrm{~ns}$ & $0.7 \pm 0.2 \mathrm{~ns}$ & $1.1 \pm 0.2 \mathrm{~ns}$ & $2.2 \pm 0.3$ \\
\hline $\begin{array}{l}77 \\
78\end{array}$ & $\begin{array}{l}\text { 2-Phenylethyl propionate } \\
\text { 2-Prenyl thanol }\end{array}$ & - $\begin{array}{l}122-70-3 \\
60-12-8\end{array}$ & $\begin{array}{l}1862 \\
1890\end{array}$ & $\begin{array}{l}\text { AR, ES } \\
\mathrm{AR}, \mathrm{OH}\end{array}$ & $\begin{array}{c}0 \\
0.2 \pm 0.04\end{array}$ & $\begin{array}{c}0 \\
362 \pm 102\end{array}$ & $\begin{array}{c}0 \\
20 \pm 2\end{array}$ & $\begin{array}{c}1884 \\
336 \pm 46\end{array}$ & $\begin{array}{l}6.3 \pm 0.7 \\
567 \pm 19\end{array}$ & $\begin{array}{c}0 \\
865 \pm 206\end{array}$ & $\begin{array}{c}1.1 \\
235 \pm 17\end{array}$ & $\begin{array}{l}1.1 \pm 0.9 \\
432 \pm 57\end{array}$ & $\stackrel{0}{814 \pm 17}$ & $\stackrel{0}{0} 271 \pm 83$ & $\begin{array}{l}172 \pm 38 \\
448 \pm 87\end{array}$ & $\begin{array}{c}0 \\
490 \pm 320\end{array}$ \\
\hline $\begin{array}{l}78 \\
79\end{array}$ & $\begin{array}{l}\text { 2-Phenyl ehhanol } \\
\text { 2-Phenylethyl butanoate }\end{array}$ & $\begin{array}{l}60-12-8 \\
103-52-6\end{array}$ & $\begin{array}{l}1890 \\
1949\end{array}$ & $\begin{array}{l}\mathrm{AR}, \mathrm{OH} \\
\mathrm{AR}, \mathrm{ES}\end{array}$ & $0.2 \pm 0.04$ & $\underset{0}{362 \pm 102}$ & ${ }_{0}^{20 \pm 2}$ & $\begin{array}{l}336 \pm 46 \\
0\end{array}$ & 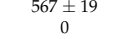 & $\frac{865 \pm 206}{0}$ & $\underset{0}{235 \pm 17}$ & $\underset{0}{432 \pm} 57$ & $\begin{array}{l}814 \pm 17 \\
0\end{array}$ & $\underset{0}{271 \pm 83}$ & $\begin{array}{l}448 \pm 87 \\
0.2 \pm 0.1\end{array}$ & $\begin{array}{l}490 \pm 320 \\
0\end{array}$ \\
\hline 80 & 2-Phenylethyl 2-methylbutanoate & 24817-51-4 & 1955 & $\begin{array}{ll}\mathrm{AR}, \mathrm{ES} \\
\mathrm{A}\end{array}$ & 0 & 0 & 0 & 0 & 0 & 0 & 0 & 0 & 0 & 0 & $4.3 \pm 1.8$ & 0 \\
\hline 83 & $\begin{array}{l}\text { Phenol }^{*} \text {-one } \\
\text { Pentadecan-2-one }\end{array}$ & $\begin{array}{l}87-66-1 \\
2345-28-0\end{array}$ & $\begin{array}{l}1919 \\
2010\end{array}$ & $\begin{array}{c}\mathrm{AR} \text {, OH } \\
\mathrm{KT}\end{array}$ & $\begin{array}{l}0.4 \pm 0.04 \\
0\end{array}$ & $\begin{array}{ll}0.38 \pm 0.01 \mathrm{~ns} \\
0\end{array}$ & $\begin{array}{l}0.5 \pm 0.1 \mathrm{~ns} \\
0\end{array}$ & - $0.15 \pm 0.01$ & $\begin{array}{l}0.07 \pm 0.03 \\
0.2 \pm 0.1\end{array}$ & $\begin{array}{c}0.4 \pm 0.1 \mathrm{~ns} \\
0\end{array}$ & $\begin{array}{l}0.5 \pm 0.1 \mathrm{~ns} \\
0\end{array}$ & $\begin{array}{l}0.2 \pm 0.1 \mathrm{~ns} \\
0\end{array}$ & $\begin{array}{l}0.6 \pm 0.2 \mathrm{~ns} \\
1.2 \pm 0.3\end{array}$ & $\begin{array}{l}0.49 \pm 0.02 \\
0.08 \pm 0.06\end{array}$ & $\begin{array}{l}0.3 \pm 0.1 \mathrm{~ns} \\
0\end{array}$ & $\begin{array}{l}0.30 \pm 0.04 \mathrm{~ns} \\
0\end{array}$ \\
\hline 84 & Octanoic acid & $124-07-2$ & 2065 & $\mathrm{FA}$ & $0.3 \pm 0.02$ & $1.0 \pm 0.2$ & 0 & $0.3 \pm 0.1 \mathrm{~ns}$ & $0.6 \pm 0.1$ & 0 & 0 & 0 & $0.27 \pm 0.03 \mathrm{~ns}$ & $0.20 \pm 0.01$ & 0 & 0 \\
\hline 85 & $\begin{array}{l}\text { 3-Methylbutyl dodecanoate } \\
\text { Hexaddoco-2-one }\end{array}$ & $\begin{array}{l}6309-51-9 \\
1877-63-8\end{array}$ & 2089 & ES & 0 & 0 & 0 & $2.3 \pm 0.4$ & $\begin{array}{c}0 \\
04+01\end{array}$ & 0 & 0 & 0 & 0 & 0 & 0 & 0 \\
\hline $\begin{array}{l}86 \\
87\end{array}$ & $\begin{array}{l}\text { Hexadecen-2-2-one } \\
\text { gamma-Decalactone }\end{array}$ & $\begin{array}{l}18787-63-8 \\
706-14-9\end{array}$ & 2128 & $\begin{array}{l}\mathrm{KT} \\
\mathrm{LA} 18\end{array}$ & $\begin{array}{l}0 \\
0 \\
0\end{array}$ & $\begin{array}{l}0 \\
0 \\
0\end{array}$ & $\begin{array}{l}0 \\
0 \\
0\end{array}$ & $\begin{array}{l}0 \\
0\end{array}$ & $\begin{array}{c}0.4 \pm 0.1 \\
0\end{array}$ & $\begin{array}{l}0 \\
0\end{array}$ & $\begin{array}{l}0 \\
0\end{array}$ & $\begin{array}{c}0 \\
1.2 \pm 0.2\end{array}$ & $\begin{array}{l}0.6 \pm 0.2 \\
5.2 \pm 1.2\end{array}$ & $\begin{array}{l}0 \\
0 \\
0\end{array}$ & $\begin{array}{c}0 \\
1.3 \pm 0.8\end{array}$ & $\begin{array}{l}0 \\
0 \\
0\end{array}$ \\
\hline 88 & 6-Pentyl-5,6 dihydro-2H-pyran-2-one & 54814-64-1 & 2222 & LA & 0 & 0 & 0 & 0 & 0 & $2.3 \pm 1.1$ & 0 & 0 & 0 & 0 & 0 & 0 \\
\hline 89 & Heptadecan-2-one & $2922-51-2$ & 2230 & $\mathrm{KT}$ & 0 & 0 & 0 & 0 & $2.1 \pm 0.6$ & 0 & 0 & 0 & 0 & 0 & 0 & 0 \\
\hline $\begin{array}{l}90 \\
91\end{array}$ & $\begin{array}{l}\text { Farnesyl acetate } \\
\text { Farnesol }\end{array}$ & $\begin{array}{l}4128-17-0 \\
4602-84-0\end{array}$ & 2287 & $\begin{array}{l}\text { TE, ES } \\
\text { TE,OH }\end{array}$ & $\begin{array}{l}0 \\
0 \\
0\end{array}$ & $\begin{array}{l}0 \\
0 \\
0\end{array}$ & $\begin{array}{l}0 \\
0 \\
0\end{array}$ & $\begin{array}{l}1.1 \pm 0.2 \\
1.7 \pm 0.3\end{array}$ & $\begin{array}{l}0 \\
0\end{array}$ & $\begin{array}{l}0 \\
0\end{array}$ & $\begin{array}{l}0 \\
0 \\
0\end{array}$ & $\begin{array}{l}0 \\
0 \\
0\end{array}$ & $\begin{array}{l}0 \\
0 \\
0\end{array}$ & $\begin{array}{l}0 \\
0 \\
0\end{array}$ & $\begin{array}{l}0 \\
0 \\
0\end{array}$ & $\begin{array}{l}0 \\
0 \\
0\end{array}$ \\
\hline 界1 & 4-Hydroxy-farinesontloxan-2-one & 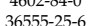 & 2456 & LA & $0_{0}^{0}$ & 0 & 0 & 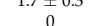 & 0 & $12+04$ & 0 & 0 & 0 & 0 & 0 & 0 \\
\hline
\end{tabular}

${ }^{1}$ Compounds indicated by star mark were excluded from multivariate analysis, ${ }^{2}$ MHMP-methyl 2-hydroxy-4-methylpentanoate, ${ }^{3}$ CAS No-chemical abstract service number ${ }^{4}$ RI-retention index (DB-Wax fused silica capillary column $30 \mathrm{~m} \times 0.25 \mathrm{~mm}$ i.d., $0.25 \mu \mathrm{m}$ film thickness), ${ }^{5}$ GR - group of a chemical compound, ${ }^{6}$ ES - ester, ${ }^{7}$ AL—aldehyde, ${ }^{8} \mathrm{OH}$-alcohol, ${ }^{9} \mathrm{KT}$-ketone, ${ }^{10}$ AR -aromatic, ${ }^{11} \mathrm{CN}$-isothiocyanoate, ${ }^{12}$ PY—pyrazine, ${ }^{13} \mathrm{FA}$-fatty acid, ${ }^{14} \mathrm{FU}$-furane, ${ }^{15}$ sulphide, ${ }^{16} \mathrm{TE}$-terpenoid, ${ }^{17} \mathrm{IM}$-imine, ${ }^{18}$ LA—lactone,

${ }^{19}$ mean \pm standard error of the mean (means are the absolute amounts expressed as areas under the chromatographic peaks and have to be read as numbers times 1000$)$, ${ }^{20}$ ns-not significantly different compare to control (Nonparametric Mann-Whitney U test, $p<0.05$ ), three different isolates of each yeast species have been used; control samples were obtained by collecting background volatiles from YPD-agar plates without yeast. 


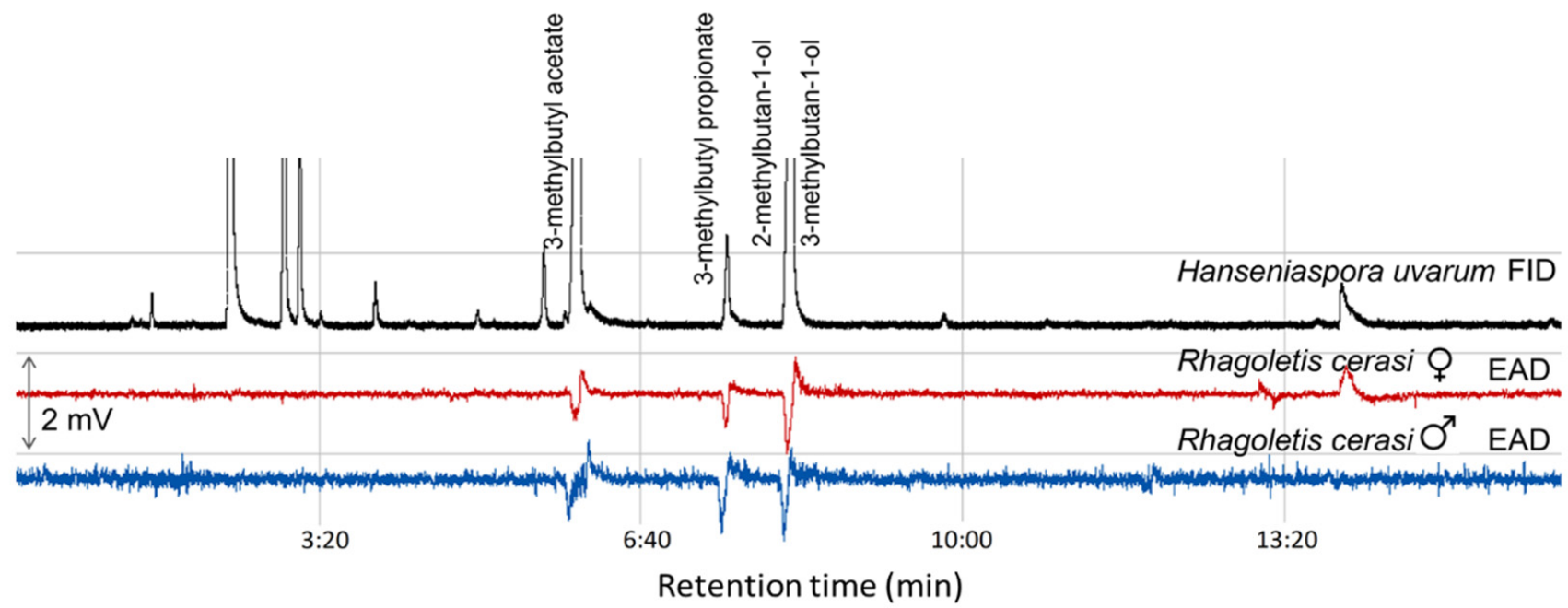

Figure 4. GC-EAD response of male and female Rhagoletis cerasi to headspace volatiles of Hanseniaspora uvarum yeast. FID, flame ionisation detector; EAD, electroantennographic detector; DB-Wax capillary column $(30 \mathrm{~m} \times 0.25 \mathrm{~mm} \times 0.25 \mu \mathrm{m}$; Agilent Technologies, Santa Clara, CA, USA.)

Table 2. Electroantennographic responses of R. cerasi flies to volatiles present in the headspace of five yeast species.

\begin{tabular}{|c|c|c|c|c|c|c|c|c|}
\hline \multirow[t]{2}{*}{ Yeast Species } & \multicolumn{2}{|c|}{ 3-MBA ${ }^{1}$} & \multicolumn{2}{|c|}{ 3-MBP 2} & \multicolumn{2}{|c|}{$2-\mathrm{MBOH}^{3}$} & \multicolumn{2}{|c|}{ 3-МBOH ${ }^{4}$} \\
\hline & Male & Female & Male & Female & Male & Female & Male & Female \\
\hline Aureobasidium pullulans & -5 & & & & $3(3)$ & $3(3)$ & $3(3)$ & $3(3)$ \\
\hline Cryptococcus wieringae & & & & & $4(4)$ & $3(3)$ & $4(4)$ & $3(3)$ \\
\hline Hanseniaspora uvarum & $4^{6}(4)^{7}$ & $4(4)$ & $4(4)$ & $3(4)$ & $4(4)$ & $4(4)$ & $4(4)$ & $4(4)$ \\
\hline Pichia kudriavzevii & $5(7)$ & $4(4)$ & $5(7)$ & $4(4)$ & $7(7)$ & $4(4)$ & $7(7)$ & $4(4)$ \\
\hline Metschnikowia pulcherrima & $1(3)$ & $3(4)$ & & & $3(3)$ & $4(4)$ & $3(3)$ & $4(4)$ \\
\hline Total & $10(14)$ & $11(12)$ & $9(11)$ & $7(8)$ & $21(21)$ & $18(18)$ & $21(21)$ & $18(18)$ \\
\hline
\end{tabular}

${ }^{1}$ 3-Methylbutyl acetate, ${ }^{2}$ 3-methylbutyl propionate, ${ }^{3}$ 2-methyl-1-butanol, ${ }^{4} 3$-methyl-1-butanol, ${ }^{5}$ compound was not detected in the headspace, ${ }^{6}$ Number of antennae responded, ${ }^{7}$ Number of antennae tested.

Unripe fruits associated with yeast-like fungi, A. pullulans, and C. wieringae yeasts produced two alcohols, 2-methyl-1-butanol and 3-methyl-1-butanol, which elicited antennae responses of $R$. cerasi flies. H. uvarum, the most common yeast species inhabiting medium-ripe fruits as well P. kudriavzevii attributed to microbiota of ripe fruits in addition to two EAD active alcohols produced two esters, 3-methybutyl acetate and 3-methylbutyl propionate, which evoked antennae responses. Ripe fruits associated yeasts, M. pulcherrima released three EAD active volatiles 3-methybutyl acetate, 2-methyl-1-butanol, and 3-methyl1-butanol (Table 2).

\subsection{EAG Dose-Response}

The antennographic responses of females to 3-methybutyl acetate and 3-methylbutyl propionate at the moderate and the higher doses were of significantly higher amplitudes compared to the responses of males (Figure $5 a, b$ ). Females showed a higher sensitivity to 2-methyl-1-butanol than males at all doses tested, and significant differences were revealed at the doses $10^{-5}, 10^{-3}$, and $10^{-2} \mathrm{mg}$ (Figure $5 \mathrm{c}$ ). Significant stronger responses of females than males were recorded to 3-methyl-1-butanol at the dose $10^{-4} \mathrm{mg}$, while antennae stimulation with the higher doses revealed stronger responses of males compared to the responses of females (Figure 5d). 


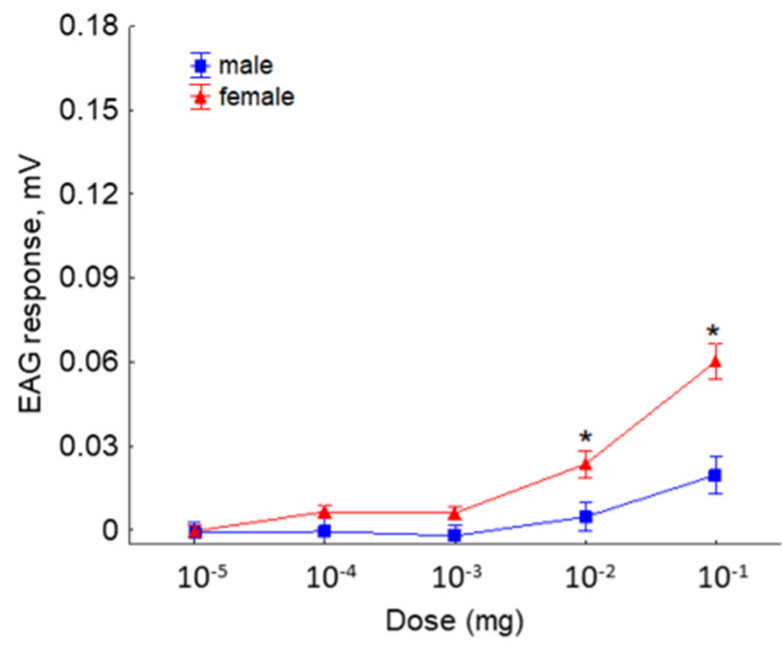

(a)

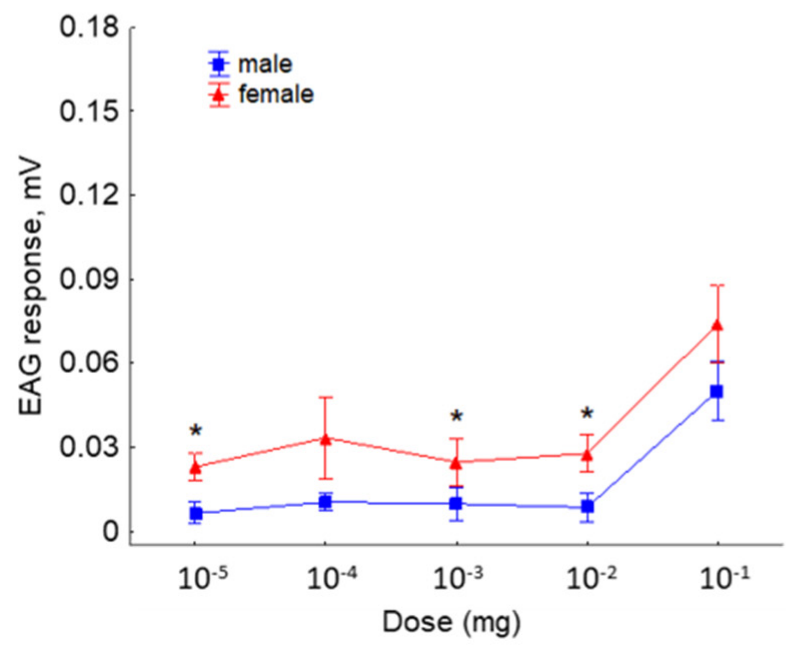

(c)

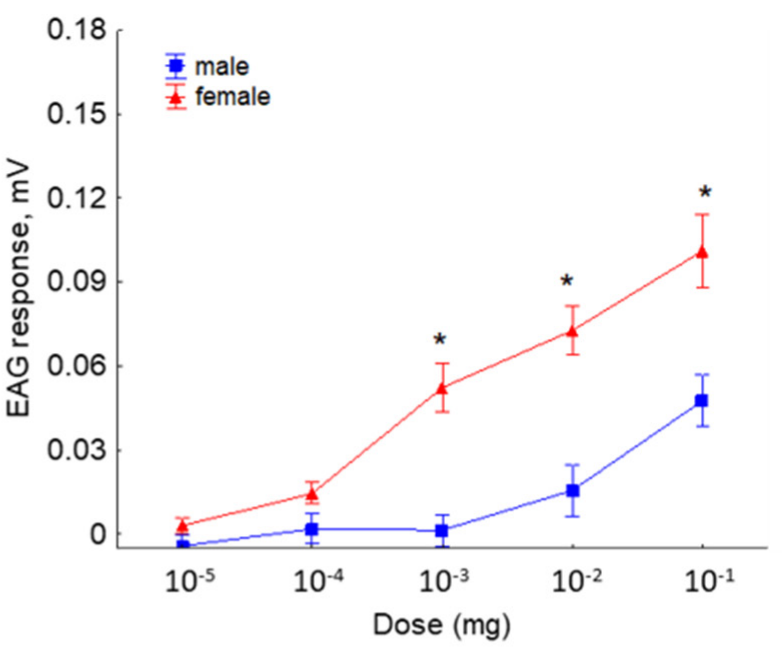

(b)

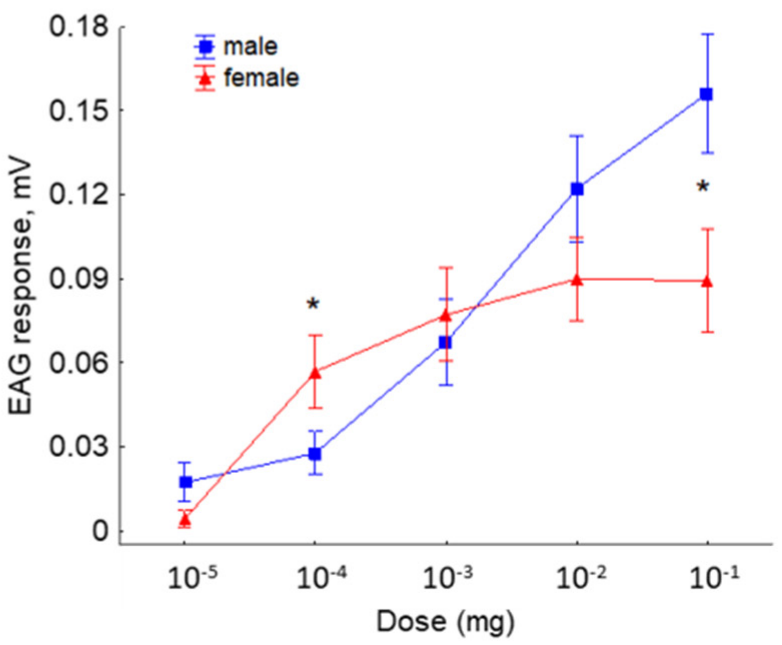

(d)

Figure 5. EAG responses (mean amplitude \pm standard error (SE), $\mathrm{mV}$ ) of $R$. cerasi male and female antennae to different doses $\left(10^{-5}\right.$ to $10^{-1} \mathrm{mg}$ ) of synthetic: (a) 3-methylbutyl acetate, (b) 3-methylbutyl propionate, (c) 2-methyl-1-butanol, and (d) 3-methyl-1-butanol. The asterisk denotes significant differences in EAG responses between sexes (paired $t$ test, $p<0.05$ ); each EAD test was replicated 13 times and each antenna used was from a different fly.

\subsection{Behavioural Tests in Olfactometer}

In the two-choice tests, $R$. cerasi males showed no preference to 3-methylbutyl acetate at the dose $10^{-2} \mathrm{mg}$, while females significantly avoided the olfactometer arm bearing 3-methylbutyl acetate at the dose $10^{-3} \mathrm{mg}$. Fruit flies of both sexes significantly preferred 3-methylbutyl propionate provided at the dose of $10^{-2} \mathrm{mg}$ for males and at the dose $10^{-3} \mathrm{mg}$ for females (Figure 6). Flies of both sexes do not discriminate between olfactory arms bearing 2-methyl-1-butanol. Males chose 3-methyl-1-butanol at the dose $10^{-2} \mathrm{mg}$ and showed no preference to a 10 times lower dose. Females preferred the alcohol at the dose of $10^{-4} \mathrm{mg}$, while a 10 times higher dose obliterated the preference. 
Males

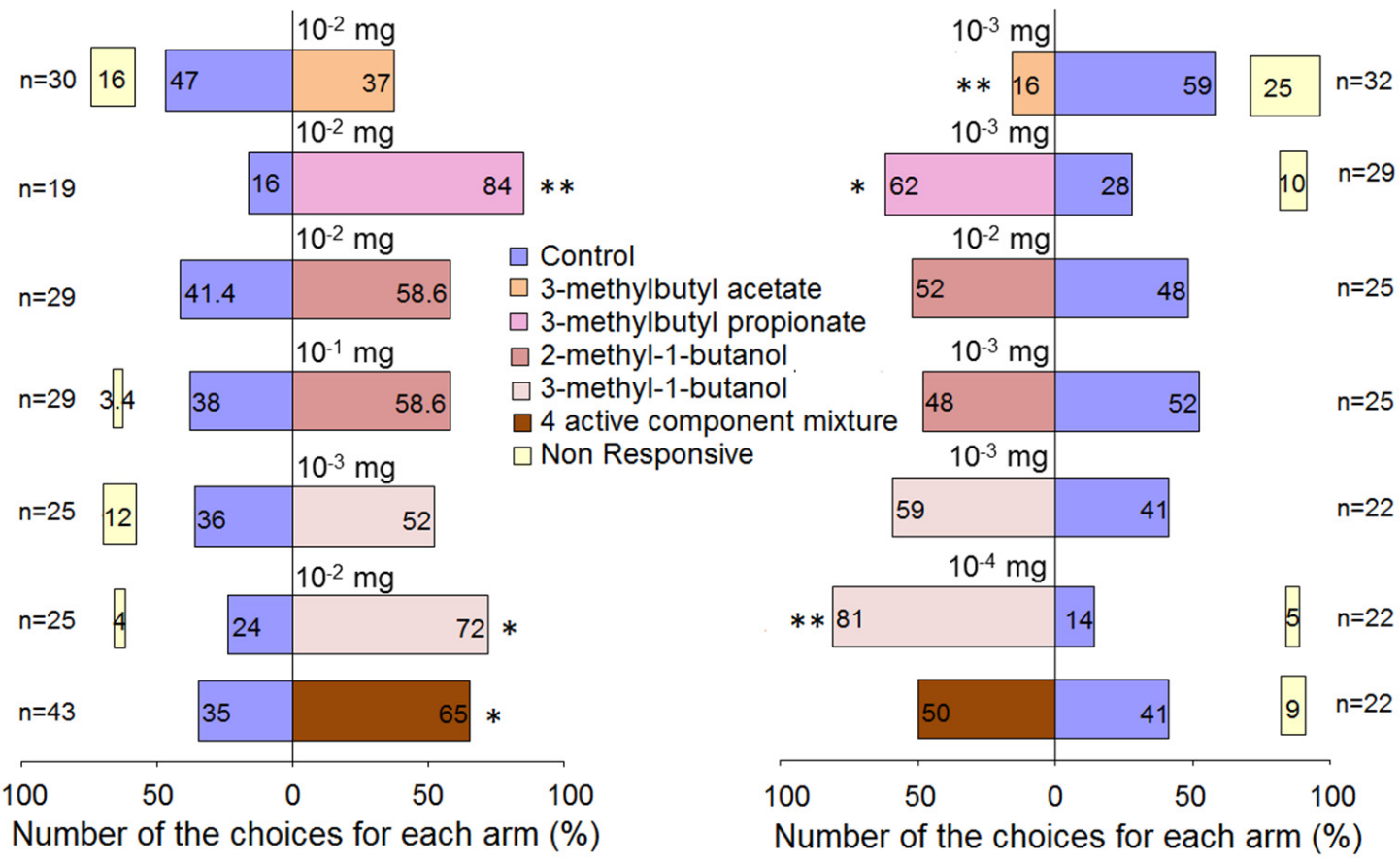

Figure 6. Behavioural responses of Rhagoletis cerasi flies in Y-tube olfactometer to EAD active volatiles. The mixture consisted of 3-methylbutyl acetate $0.55 \mathrm{mg}$, 3-methylbutyl propionate $0.05 \mathrm{mg}$, 2-methyl1-butanol $0.012 \mathrm{mg}$, and 3-methyl-1-butanol $0.28 \mathrm{mg}$. n-number of flies tested. Stars denote significant differences in behavioural responses of flies between stimulus and control: ${ }^{*} p<0.05$ and ${ }^{* *} p<0.01$ by $\chi^{2}$ test.

\section{Discussion}

Yeasts produce a wide range of volatile compounds [19]. Common volatiles at large amounts emitted by different yeast species originate from primary metabolism pathways and these compounds are considered as side products or waste compounds [20]. Ethanol and ethyl acetate are the best-known examples of such volatile metabolites. Our analysis showed that yeasts of $S$. paradoxus species released high amounts of ethanol while P. anomala, P. kudriavzevii, H. uvarum, and M. pulcherrima yeasts produced the largest amounts of ethyl acetate, an ester derived from ethanol. Ethanol or ethyl acetate were the major components in the volatile bouquets of these yeast species. Interestingly, we did not detect ethanol and ethyl acetate in the headspaces of P. kluyveri and P. membranifaciens yeasts. More structurally diverse volatiles released by yeasts are derived from amino or fatty acid synthesis and degradation as well as from terpene biosynthetic pathways [19,20]. Analysis of volatile profiles of eleven yeast species isolated from sweet and sour cherry fruits showed that esters and alcohols were the most numerous groups of volatiles.

The multidimensional scaling analysis revealed that volatile blends of all yeast species were clearly separated from each other, therefore, the amount and composition of volatiles characterised yeast species. Our results are consistent with previous findings that volatile profiles of microorganisms are species-specific, reflecting specific metabolic activities of the particular microorganisms [19].

The ecological role of microbial volatiles falls in two major groups: they are important as semiochemicals mediating information flow at intra- and inter-specific levels; and function as promoters or inhibitors of microbial growth [21-23]. Yeast volatiles play an essential role in distribution of yeast from one habitat to another by attracting vector insects. Our data showed that 3-methybutyl acetate, 3-methylbutyl propionate, 2-methyl-1butanol, and 3-methyl-1-butanol emitted by yeasts populating P. avium and P. cerasus fruits elicited electroantennographic responses in both sexes of $R$. cerasi fruit flies, a common 
pest of cherry fruits. Recently, it was reported that ten volatiles emitted by P. kudriavzevii yeast species including 3-methybutyl acetate, 3-methylbutyl propionate, and 3-methyl-1butanolelicited antennal responses in males and females of closely related $R$. batava flies while 2-methyl-1-butanol was not EAD active [24].

Females showed the higher sensitivity to 3-methybutyl acetate, 3-methylbutyl propionate, and 2-methyl-1-butanol than males and the more pronounced difference in antennal sensitivity was recorded at higher doses of both esters. As far as we know, higher antennae sensitivity of females to microbial volatiles has not been reported in other tephritid fruit flies.

Two EAD active compounds 3-methylbutyl propionate and 3-methyl-1-butanol stimulated both sexes of $R$. cerasi flies to choose the olfactometer arm bearing these volatiles versus control without stimulus, while 2-methyl-1-butanol did not significantly affect the choice of flies. 3-Methyl-1-butanol is the end product of degradation of the amino acid leucine [20] and is found in volatile bouquets of many yeast species [20,25]. In our samplings, 3-methyl-1-butanol at various amounts was isolated from the emissions of all eleven yeast species. As an attractant, 3-methyl-1-butanol functions in five dipteran species [26] including one tephritid species [25]. Attractiveness of 3-methylbutyl propionate has not been reported for any dipteran species.

The two-choice test showed that $R$. cerasi females avoided the olfactometer hand bearing 3-methybutyl acetate at the dose $10^{-2} \mathrm{mg}$, while males did not significantly discriminate stimulus versus the control at even higher $10^{-1} \mathrm{mg}$ dose. $R$. cerasi females prefer to lay eggs in cherries at the stage of colour change from green to yellow [27] (i.e., just at the beginning of ripening). At the early maturation stage of fruits, yeast-like fungi from Aureobasidium genus, yeasts from Cryptococcus, Taphrina, Cladosporium, and some other genus are more prevalent compared to yeast from Pichia, Metschnikowia, Saccharomyces, and Torulaspora genus inhabiting ripe fruits $[3,7,28]$. Analysis of chromatographic profiles of yeast emitted volatiles revealed that unripe fruits associated with yeast-like fungi, Aureobasidium pullulans, and Cryptococcus wieringae yeasts produced none or very low amounts of 3-methybutyl acetate, acting as a repellent to $R$. cerasi females. 3-Methybutyl acetate is a ubiquitous volatile in the odour bouquets of ripe and fermenting fruit-yeast complexes [29], which is in agreement with our data showing that fermenting yeasts emitted large amounts of this acetate. Contrary to R. cerasi, many Drosophila species oviposit in ripe fruits and berries and 3-methybutyl acetate released by ripe fruits associated yeasts functions as an attractant $[29,30]$.

Ammonium acetate is reported as the most efficient food attractant for $R$. cerasi flies [31]. The number of yeast-based commercially available attractive lures such as brewer's yeast waste, baker's yeast (Saccharomyces cerevisiae), and Torula yeast (Candida utilis) have been used in tephritid pest control programs [32], however, none of the formulations tested showed a potential to control R. cerasi fruit flies. A possible explanation is that all lures released high amounts of 3-methybutyl acetate, the repellent to $R$. cerasi fruit flies. Our data suggest that yeast species selected for an efficient lure to target $R$. ceras $i$ pests should release high amounts of 3-methylbutyl propionate and / or 3-methyl-1-butanol and do not emit 3-methybutyl acetate. Moreover, our data provide a background for the application of behaviour modifying semiochemicals in push-pull and other integrated pest management techniques to control $R$. cerasi fruit flies.

\section{Conclusions}

The odour blends emitted by yeasts were species-specific. 3-Methybutyl acetate, 3-methylbutyl propionate, 3-methylbutanol, and 2-methyl-1-butanol released by yeasts populating $P$. avium and $P$. cerasus fruits elicited electroantennographic responses and modulated behaviour of $R$. cerasi fruit flies, a common pest of cherry fruits. Therefore, these olfactory and behaviourally active compounds show potential for use in integrated pest management techniques to control $R$. cerasi fruit flies. 
Author Contributions: Conceptualisation, R.M., E.S. and V.B.; Methodology, D.A., S.R., V.A. and R.S.; Software, R.M., S.R. and L.B.-Č.; Validation, V.A., S.R., L.B.-Č., E.S. and R.M.; Formal analysis, V.A., S.R., J.B., L.B.-Č., E.S. and R.M.; Investigation, D.A., S.R., V.A., R.S. and L.B.-Č.; Resources, R.M., V.B. and E.S.; Data curation, V.A., S.R., L.B.-Č. and E.S.; writing-original draft preparation, R.M.; writing-review and editing, R.M., S.R., L.B.-Č., V.B. and E.S.; visualisation, R.M., S.R. and L.B.-Č.; Supervision, R.M. and V.B.; Project administration, R.M. and L.B.-Č.; Funding acquisition, R.M. All authors have read and agreed to the published version of the manuscript.

Funding: This research was funded by EUROPEAN SOCIAL FUND, grant number 09.3.3-LMT-K712-01-0099 under grant agreement with the Research Council of Lithuania (LMTLT).

Institutional Review Board Statement: Not applicable.

Informed Consent Statement: Not applicable.

Data Availability Statement: Not applicable.

Acknowledgments: We thank A. Amšiejus and L. Levulytė for the kind permission to collect puparia in their cherry orchards. Thanks are also given to I. Vepštaitè-Monstavičè and J. Lukša for providing cherry fruits.

Conflicts of Interest: The authors declare no conflict of interest. The funders had no role in the design of the study; in the collection, analyses, or interpretation of data; in the writing of the manuscript, or in the decision to publish the results.

\section{Appendix A}

Table A1. List of yeast isolates analysed in this study.

\begin{tabular}{|c|c|c|c|}
\hline Yeast Species & Strain & GenBank Reference & Identity (\%) \\
\hline \multirow{3}{*}{ Aureobasidium pullulans } & PA-4-13 & MN400109 & 100 \\
\hline & PC-5-28 & HQ909088 & 99.6 \\
\hline & PC-4-8 & MW361317 & 99.8 \\
\hline \multirow{3}{*}{ Cryptococcus wieringae } & PC-4-6 & KF981864 & 100 \\
\hline & PC-4-9 & KF981864 & 100 \\
\hline & PA-5-41 & KF981864 & 100 \\
\hline \multirow{3}{*}{ Metschnikowia pulcherrima } & PC-4-5 & MF574308 & 100 \\
\hline & PA-5-13 & КТ029787 & 99.7 \\
\hline & PA-5-47 & MK352050 & 100 \\
\hline \multirow{3}{*}{ Hanseniaspora uvarum } & PA-5-27 & MF062209 & 100 \\
\hline & PC-3-8 & MK352020 & 99.9 \\
\hline & PC-5-12 & KY103573 & 100 \\
\hline \multirow{3}{*}{ Pichia kudriavzevii } & PA-1-15 & MF685423 & 100 \\
\hline & PC-4-25 & MF685411 & 100 \\
\hline & PC-4-19.3 & MG015972 & 99.6 \\
\hline \multirow{3}{*}{ Pichia fermentans } & PC-5-47 & FJ713081 & 100 \\
\hline & PC-4-19.1 & MF462777 & 100 \\
\hline & PA-4-39 & KY104537 & 100 \\
\hline \multirow{3}{*}{ Pichia kluyveri } & PA-4-30 & JX103190 & 100 \\
\hline & PA-4-34 & KY108823 & 99.8 \\
\hline & PC-4-35 & KC510043 & 100 \\
\hline \multirow{3}{*}{ Pichia membranifaciens } & PC-3-36 & JX188207 & 100 \\
\hline & PC-2-71 & FJ231461 & 100 \\
\hline & PA-2-55 & JX188207 & 99.6 \\
\hline \multirow{3}{*}{ Saccharomyces paradoxus } & PA-5-14 & FJ713072 & 100 \\
\hline & PC-3-33 & FJ713072 & 100 \\
\hline & PC-3-59 & KY105204 & 99.9 \\
\hline
\end{tabular}


Table A1. Cont.

\begin{tabular}{cccc}
\hline Yeast Species & Strain & GenBank Reference & Identity (\%) \\
\hline \multirow{2}{*}{ Torulaspora delbrueckii } & PA-4-16 & KY105641 & 100 \\
& PA-5-17 & MN371902 & 99.8 \\
& PC-2-42 & MK352012 & 100 \\
Pichia anomala & PC-5-5 & KJ527050 & 100 \\
& PA-5-18 & MH248067 & 100 \\
\hline
\end{tabular}

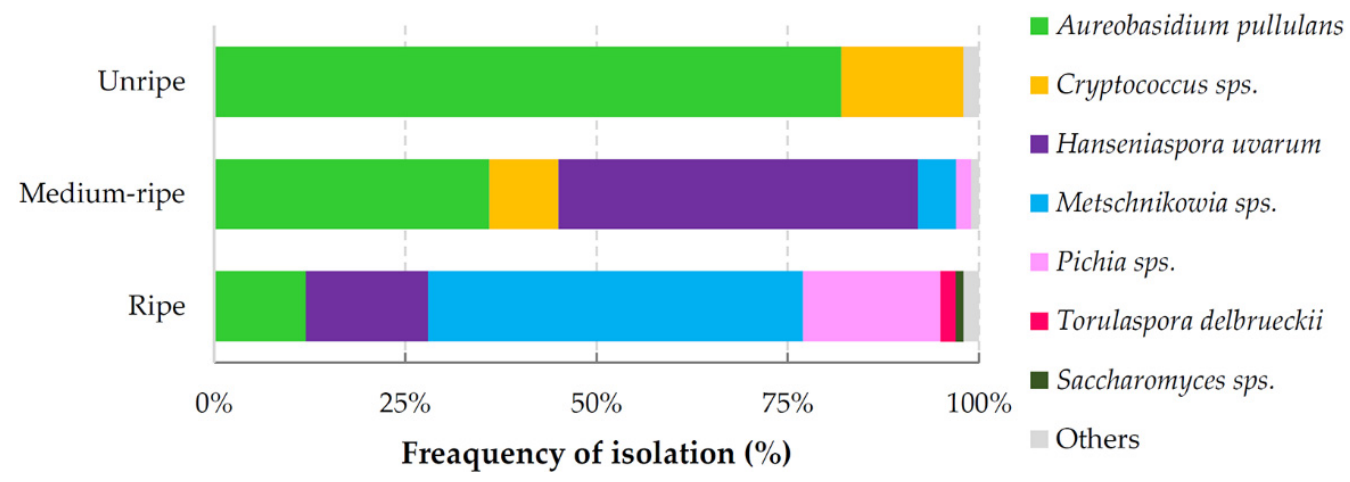

Figure A1. Frequency of yeast isolation among cherry samples at different ripening stages. C. wieringae dominated among Cryptococcus genus species; M. pulcherrima frequently occurred among Metschnikowia yeasts; Pichia genus were represented by P. anomala, P. kudriavzevii, P. kluyveri, P. fermentans, and P. membranifaciens yeasts; S. paradoxus occurred among Saccharomyces yeasts; others represented yeasts occurred in low frequency.

\section{References}

1. Starmer, W.T.; Lachance, M.-A. Chapter 6-Yeast Ecology. In The Yeasts, 5th ed.; Kurtzman, C.P., Fell, J.W., Boekhout, T., Eds.; Elsevier: London, UK, 2011; pp. 65-83. [CrossRef]

2. Allard, S.M.; Ottesen, A.R.; Brown, E.W.; Micallef, S.A. Insect exclusion limits variation in bacterial microbiomes of tomato flowers and fruit. J. Appl. Microbiol. 2018, 125, 1749-1760. [CrossRef] [PubMed]

3. Barata, A.; Malfeito-Ferreira, M.; Loureiro, V. The microbial ecology of wine grape berries. Int. J. Food Microbiol. 2012, 153, 243-259. [CrossRef] [PubMed]

4. Singh, P.; Gobbi, A.; Santoni, S.; Hansen, L.H.; This, P.; Peros, J.P. Assessing the impact of plant genetic diversity in shaping the microbial community structure of Vitis vinifera phyllosphere in the Mediterranean. Front. Life Sci. 2018, 11, 35-46. [CrossRef]

5. Vepstaite-Monstavic, I.; Luksa, J.; Staneviclene, R.; Strazdaite-Zieliene, Z.; Yurchenko, V.; Serva, S.; Serviene, E. Distribution of apple and blackcurrant microbiota in Lithuania and the Czech Republic. Microbiol. Res. 2018, 206, 1-8. [CrossRef] [PubMed]

6. Kioroglou, D.; Kraeva-Deloire, E.; Schmidtke, L.M.; Mas, A.; Portillo, M.C. Geographical origin has a greater impact on grape berry fungal community than grape variety and maturation state. Microorganisms 2019, 7, 669. [CrossRef]

7. Luksa, J.; Vepstaite-Monstavice, I.; Apsegaite, V.; Blazyte-Cereskiene, L.; Staneviciene, R.; Strazdaite-Zieliene, Z.; Ravoityte, B.; Aleknavicius, D.; Buda, V.; Mozuraitis, R.; et al. Fungal microbiota of sea buckthorn berries at two ripening stages and volatile profiling of potential biocontrol yeasts. Microorganisms 2020, 8, 456. [CrossRef]

8. Stefanini, I. Yeast-insect associations: It takes guts. Yeast 2018, 35, 315-330. [CrossRef] [PubMed]

9. Blackwell, M. Made for each other: Ascomycete yeasts and insects. Microbiol. Spectr. 2017, 5, 18. [CrossRef] [PubMed]

10. Janson, E.M.; Stireman, J.O.; Singer, M.S.; Abbot, P. Phytophagous insect-microbe mutualisms and adaptive evolutionary diversification. Evolution 2008, 62, 997-1012. [CrossRef]

11. Dobzhansky, T.; Cooper, D.M.; Phaff, H.J.; Knapp, E.P.; Carson, H.L. Studies on the ecology of Drosophila in the Yosemite region of California Differential attraction of species of Drosophila to different species of yeasts. Ecology 1956, 37, 544-550. [CrossRef]

12. Date, P.; Dweck, H.K.M.; Stensmyr, M.C.; Shann, J.; Hansson, B.S.; Rollmann, S.M. Divergence in olfactory host plant preference in D-mojavensis in response to cactus host use. PLoS ONE 2013, 8, 10. [CrossRef] [PubMed]

13. Keesey, I.W.; Knaden, M.; Hansson, B.S. Olfactory specialization in Drosophila suzukii supports an ecological shift in host preference from rotten to fresh fruit. J. Chem. Ecol. 2015, 41, 121-128. [CrossRef]

14. Scheidler, N.H.; Liu, C.; Hamby, K.A.; Zalom, F.G.; Syed, Z. Volatile codes: Correlation of olfactory signals and reception in Drosophila-yeast chemical communication. Sci. Rep. 2015, 5, 14059. [CrossRef]

15. Holighaus, G.; Rohlfs, M. Fungal allelochemicals in insect pest management. Appl. Microbiol. Biotechnol. 2016, 100, 5681-5689. [CrossRef] 
16. Staneviciene, R.; Luksa, J.; Strazdaite-Zieliene, Z.; Ravoityte, B.; Losinska-Siciuniene, R.; Mozuraitis, R.; Serviene, E. Mycobiota in the carposphere of sour and sweet cherries and antagonistic features of potential biocontrol yeasts. Microorganisms 2021, 9, 1423. [CrossRef] [PubMed]

17. Buda, V.; Blazyte-Cereskiene, L.; Radziute, S.; Apsegaite, V.; Stamm, P.; Schulz, S.; Aleknavicius, D.; Mozuraitis, R. Male-produced (-)-delta-heptalactone, pheromone of fruit fly Rhagoletis batava (Diptera: Tephritidae), a sea buckthorn berries pest. Insects 2020, 11, 138. [CrossRef] [PubMed]

18. Hare, D.J. Bioassay methods with terrestrial invertebrates. In Methods in Chemical Ecology: Bioassay Methods; Millar, J.G., Haynes, K.F., Eds.; Kluwer Academic Publishers: Norwell, MA, USA, 2000; pp. 212-270.

19. Lemfack, M.C.; Nickel, J.; Dunkel, M.; Preissner, R.; Piechulla, B. mVOC: A database of microbial volatiles. Nucleic Acids Res. 2014, 42, D744-D748. [CrossRef] [PubMed]

20. Ebert, B.E.; Halbfeld, C.; Blank, L.M. Exploration and Exploitation of the Yeast Volatilome. Curr. Metab. 2017, 5, 102-118. [CrossRef]

21. Schmidt, R.; Cordovez, V.; de Boer, W.; Raaijmakers, J.; Garbeva, P. Volatile affairs in microbial interactions. ISME J. 2015, 9 , 2329-2335. [CrossRef]

22. Becher, P.G.; Hagman, A.; Verschut, V.; Chakraborty, A.; Rozpedowska, E.; Lebreton, S.; Bengtsson, M.; Flick, G.; Witzgall, P.; Piskur, J. Chemical signaling and insect attraction is a conserved trait in yeasts. Ecol. Evol. 2018, 8, 2962-2974. [CrossRef]

23. Kai, M.; Haustein, M.; Molina, F.; Petri, A.; Scholz, B.; Piechulla, B. Bacterial volatiles and their action potential. Appl. Microbiol. Biotechnol. 2009, 81, 1001-1012. [CrossRef]

24. Mozuraitis, R.; Aleknavicius, D.; Vepstaite-Monstavice, I.; Staneviciene, R.; Emami, S.N.; Apsegaite, V.; Radziute, S.; Blazyte-Cereskiene, L.; Serviene, E.; Buda, V. Hippophae rhamnoides berry related Pichia kudriavzevii yeast volatiles modify behaviour of Rhagoletis batava flies. J. Adv. Res. 2020, 21, 71-77. [CrossRef] [PubMed]

25. Vitanovic, E.; Aldrich, J.R.; Boundy-Mills, K.; Cagalj, M.; Ebeler, S.E.; Burrack, H.; Zalom, F.G. Olive Fruit Fly, Bactrocera oleae (Diptera: Tephritidae), attraction to volatile compounds produced by host and insect-associated yeast strains. J. Econ. Entomol. 2020, 113, 752-759. [CrossRef] [PubMed]

26. Davis, T.S.; Landolt, P.J. A survey of insect assemblages responding to volatiles from a ubiquitous fungus in an agricultural landscape. J. Chem. Ecol. 2013, 39, 860-868. [CrossRef]

27. Daniel, C.; Grunder, J. Integrated management of European cherry fruit fly Rhagoletis cerasi (L.): Situation in Switzerland and Europe. Insects 2012, 3, 956-988. [CrossRef]

28. Ghanbarzadeh, B.; Sampiao, J.P.; Arzanlou, M. Grape maturity significantly influences yeast community on grape berries: Basidiomycetous yeasts are dominant colonizers of immature grape berries in northwestern Iran. Nova Hedwig. 2021, 113, 191-206. [CrossRef]

29. Revadi, S.; Vitagliano, S.; Stacconi, M.V.R.; Ramasamy, S.; Mansourian, S.; Carlin, S.; Vrhovsek, U.; Becher, P.G.; Mazzoni, V.; Rota-Stabelli, O.; et al. Olfactory responses of Drosophila suzukii females to host plant volatiles. Physiol. Entomol. 2015, 40, 54-64. [CrossRef]

30. Cha, D.H.; Gill, M.A.; Epsky, N.D.; Werle, C.T.; Adamczyk, J.J.; Landolt, P.J. From a non-target to a target: Identification of a fermentation volatile blend attractive to Zaprionus indianus. J. Appl. Entomol. 2015, 139, 114-122. [CrossRef]

31. Katsoyannos, B.I.; Papadopoulos, N.T.; Stavridis, D. Evaluation of trap types and food attractants for Rhagoletis cerasi (Diptera: Tephritidae). J. Econ. Entomol. 2000, 93, 1005-1010. [CrossRef]

32. Biasazin, T.D.; Chernet, H.T.; Herrera, S.L.; Bengtsson, M.; Karlsson, M.F.; Lemmen-Lechelt, J.K.; Dekker, T. Detection of volatile constituents from food lures by Tephritid fruit flies. Insects 2018, 9, 119. [CrossRef] 